\title{
Unstructured Grid Adaptation and Solver Technology for Turbulent Flows
}

\author{
Michael A. Park* \\ NASA Langley Research Center, Hampton, VA 23681, USA \\ Nicolas Barral ${ }^{\dagger}$ \\ Imperial College London, South Kensington Campus, London SW7 2AZ, UK \\ Daniel Ibanez \\ Sandia National Laboratories, P.O. Box 5800, Albuquerque, NM 87185-1321, USA \\ Dmitry S. Kamenetskiy ${ }^{\S}$ \\ Boeing Commercial Airplanes, Seattle, WA, USA \\ Joshua A. Krakos ${ }^{\mathrm{I}}$ and Todd Michal ${ }^{\mathrm{II}}$ \\ The Boeing Company, St. Louis, MO, USA \\ Adrien Loseille** \\ INRIA Paris-Saclay, Alan Turing Building, 91120 Palaiseau, France
}

\begin{abstract}
Unstructured grid adaptation is a tool to control Computational Fluid Dynamics (CFD) discretization error. However, adaptive grid techniques have made limited impact on production analysis workflows where the control of discretization error is critical to obtaining reliable simulation results. Issues that prevent the use of adaptive grid methods are identified by applying unstructured grid adaptation methods to a series of benchmark cases. Once identified, these challenges to existing adaptive workflows can be addressed. Unstructured grid adaptation is evaluated for test cases described on the Turbulence Modeling Resource (TMR) web site, which documents uniform grid refinement of multiple schemes. The cases are turbulent flow over a Hemisphere Cylinder and an ONERA M6 Wing. Adaptive grid force and moment trajectories are shown for three integrated grid adaptation processes with Mach interpolation control and output error based metrics. The integrated grid adaptation process with a finite element (FE) discretization produced results consistent with uniform grid refinement of fixed grids. The integrated grid adaptation processes with finite volume schemes were slower to converge to the reference solution than the FE method. Metric conformity is documented on grid/metric snapshots for five grid adaptation mechanics implementations. These tools produce anisotropic boundary conforming grids requested by the adaptation process.
\end{abstract}

\section{Introduction}

The use of Reynolds-averaged Navier-Stokes (RANS) with a turbulence model has become a critical tool for the design of aerospace vehicles. However, the issues that affect the grid convergence of three dimensional (3D) configurations are not completely understood, as documented in the AIAA Drag Prediction Workshop series [1--3]. To identify and address the issues preventing grid convergence, a series of special sessions has been organized for the Evaluation of RANS Solvers on Benchmark Aerodynamic Flows [4]. This evaluation includes 2D test cases [5] and 3D

\footnotetext{
* Research Scientist, Computational AeroSciences Branch, AIAA Associate Fellow.

$\dagger$ Research Associate, Department of Earth Science and Engineering.

$\doteqdot$ Research Scientist.

${ }^{\S}$ Engineer, AIAA Senior Member.

"Engineer, AIAA Senior Member.

"Technical Fellow, AIAA Senior Member.

**Researcher, GAMMA3 Team, AIAA Member.
} 
test cases [6]. This effort uses a set of 3D test cases described on the Turbulence Modeling Resource (TMR) Website [7] as 3D Hemisphere Cylinder (new) and 3D ONERA M6 Wing with the Spalart-Allmaras (SA) turbulence model [8].

Alauzet and Loseille [9] documented the dramatic progress made in the last decade for solution-adaptive methods that includes the anisotropy to resolve simulations with shocks and boundary layers. Remaining challenges are identified by the application of solution-adaptive techniques to complex simulations. Park et al. [10] documented the current state of solution-based anisotropic grid adaptation and motivated further development with the impacts that improved capability would have on aerospace analysis and design in the broader context of the CFD Vision 2030 Study by Slotnick et al. [11]. The Vision Study provides a number of case studies to illustrate the current state of CFD capability and capacity and the potential impact of emerging High Performance Computing (HPC) environments forecast in the year 2030 .

The evaluation of these benchmark RANS cases is a continuation of the efforts of Park et al. [12] to decompose the solution-adaptive process into a number of subprocesses that can be independently verified, evaluated, and improved. Developing and documenting the evaluation methods is equally important as the test cases themselves. The informal Unstructured Grid Adaptation Working Group (UGAWG) has been formed to continue this process as described in their first benchmark [13], which focused on evaluating adaptive grid mechanics for analytic metric fields on planar and simple curved domains. This first benchmark contains a list of future directions, which includes the focus of this paper: solution-driven adaptation.

In this work, the metric tensor fields are based on the Mach fields of discrete solutions with (nominally second-order) noise. In addition, discrete Hessian reconstruction is used to transform the Mach field into a metric tensor. This solution-based metric controls the $L_{p}$ norm of Mach interpolation error [14], which may result in slower convergence of forces and moments as compared to output-based (goal-based) error estimates. The TMR provides the results of multiple codes on a set of uniformly-refined grids to evaluate the convergence of integrated forces. The goal of this paper is to evaluate the grid adaptive process in the context of these uniformly-refined grids, where the $L_{p}$ metric has the advantage of simplicity and implementation in multiple codes for comparison. Output-based approaches are also included to study their impact on the convergence of integrated forces. Implementation details of adaptive grid mechanics are evaluated by examining the metric conformity statistics produced by adapting grid-metric snapshots from the adaptive process. The same evaluation methods of the first UGAWG benchmark [13] are used on these adapted grid and interpolated metric pairs, which include edge length and element shape descriptive statistics related to metric conformity.

A central repository for the UGAWG has been established on GitHub in a group account github.com/UGAWG. Information required to set up and run benchmark cases [13] is available in adapt-benchmarks. The resulting grids and descriptive statistics from the application of multiple adaptation tools to this benchmark is available in adapt-results. The geometry, initial grids, boundary conditions, and reference conditions for the solution-adaptive cases are in solution-adapt-cases. The Computer Aided Drafting (CAD) models were developed in the Electronic Geometry Aircraft Design System (EGADS [15]) and exported in STEP (Standard for the Exchange of Product model data) and IGES (Initial Graphics Exchange Specification) formats. Adapted grids, Mach fields, and metric fields are in solution-adapt-results.

The Gamma Mesh Format is used for grid, geometry association, solution, and metric interchange. A reference implementation of readers and writers with documentation is available at github.com/LoicMarechal/libMeshb. The grid is stored with Vertices, Triangles, and Tetrahedra keywords, where the id of Tetrahedra should be zero and the id of Triangles should be the one-based face index of the supporting geometry face. The Mach and metric fields use SolAtVertices keywords with one field for scalars and matrices, respectively.

The standard practice of reconstructing the grid association to the topology and parameters of the CAD model is error prone. The information describing this association can be persisted to eliminate the possibility of CAD association error. The grid generation and adaptation community has not adopted a standard, but defining a common standard would benefit grid generation and adaptation process. In the UGAWG repositories, geometry association is optionally included as VerticesOnGeometricVertices, VerticesOnGeometricEdges, VerticesOnGeometricTriangles, and Edges keywords. Edges have the one-based edge index id of the supporting geometry edge. The discrete VerticesOnGeometricVertices have the one-based node index id of the supporting topological geometry node. VerticesOnGeometricEdges have the parametric $t$ value of the supporting geometry edge and the projection distance (typically zero if the geometry is evaluated). VerticesOnGeometricTriangles have the parametric $u$ and $v$ values of the supporting geometry face and the projection distance. 


\section{Grid Adaptation Mechanics for Metric Conformity}

UGAWG members provided grid adaptation codes that are the result of industry, academic, and government investment and development. Some are open source, which allows for detailed examination of implementation details. These codes take an existing grid as input and apply modifications to obtain a new grid that is better aligned with a given metric. These codes try to output a unit grid, i.e., a grid in which edge lengths as measured by the metric distance (see Eq. (1) are close to one. The following subsections give an overview of each code.

\section{A. EPIC}

The EPIC anisotropic grid adaptation process provides a modular framework for anisotropic unstructured grid adaptation that can be linked with external flow solvers. EPIC relies on repeated application of edge break, edge collapse, and element reconnection operations to modify a grid such that element edge lengths match a given anisotropic metric tensor field. EPIC-ICS uses only edge insertion, edge collapse, and element swaps. EPIC-ICS is used exclusively for the solution-adaptive results. The metric conformity section also includes the EPIC-ICSM variant, which adds node movement to the algorithm to produce peaked metric conformity statistics at the expense of increased execution time.

The metric field on the adapted grid is continuously interpolated from the initial metric field. Several methods are available to preprocess the metric so as to limit minimum and maximum local grid sizes, control stretching rates of grid size and/or anisotropy, and ensure smoothness of the resulting distribution. In addition, the metric distribution can be limited relative to the initial grid and/or to the local geometry surface curvature. The surface grid is maintained on an IGES geometry definition with geometric projections and a local regriding. The adaptive grid mechanics are applied to the tetrahedral grid with the option to insert right angle prismatic or tetrahedral elements into the adapted grid near wall boundaries. Adding a near wall boundary grid has the additional benefit of accelerating refinement of the adapted grid normal to the wall.

\section{B. refine}

The refine open source grid adaptation mechanics package was developed by NASA. It is available via github. Com/NASA/refine under the Apache License, Version 2.0. It is designed to output a unit grid [16] in a provided metric field. The current version under development uses the combination of edge split and collapse operations proposed by Michal and Krakos [17]. Node relocation is performed to improve adjacent element shape. A new ideal node location of the node is created for each adjacent element. A convex combination of these ideal node locations is chosen to yield a new node location update that improves the element shape measure in the anisotropic metric [18]. Geometry is accessed through the EGADS application program interface.

\section{Omega_h}

Omega_h github.com/ibaned/omega_h is an open-source grid adaptation library [19, 20], developed by Rensselaer Polytechnic Institute and subsequently by Sandia National Laboratories. Like the other codes in this study, it aims to be a state-of-the-art implementation of grid adaptation by local topological modifications. Omega_h has certain unique objectives: First, it targets tightly coupled adaptivity within a simulation, which requires remapping the solution accurately. This motivates minimizing the number of modifications. Second, it targets simulations outside the CFD space, including solid mechanics and shock hydrodynamics. This motivates a much stronger focus on element shape and efficient operation with isotropic metrics. Third, it targets high performance execution using threading and Graphics Processing Units (GPUs).

The core algorithm in Omega_h consists of one loop of alternating edge splitting and edge collapsing to satisfy length, followed by another loop that uses edge swapping and edge collapsing to improve element shape. Snapping to geometry (using EGADS) is part of the second (element shape) loop. All nodes are moved as far as they can toward the snapping goal while maintaining valid element volumes. Swapping and collapsing are used to correct shapes and snapping is resumed. The snapping and element shape improvement loop continues until the nodes are on the EGADS geometry.

Omega_h handles highly anisotropic metrics with an iterative approach. During each iteration, Omega_h selects an interpolated metric that is between the target and the implied metric of the current grid, then applies its full adaptive algorithm. After several cycles, Omega_h approaches the highly anisotropic target metric. In both snapping and metric conformity, the criteria that determines the step size is element shape (the step is halved until all elements are above a shape measure limit in the interpolated metric space). For all results presented, this limit is 0.3 (see Eq. (3)). 


\section{Pragmatic}

Pragmatic [21] meshadaptation.github.io is an open source 2D and 3D anisotropic adaptation code developed as a $\mathrm{C}++$ library at Imperial College London. Initially targeted at geophysical flow simulations, Pragmatic aims at generating quality grids for a wide range of numerical simulations. It has been integrated with the PETSc library [22, 23].

The input grid is modified through a series of local grid manipulations. First, iterative applications of coarsening (edge collapse), edge/face swapping and refinement (edge splitting) is used to optimize the resolution and the quality of the grid. Second, an element-shape-constrained Laplacian smoothing step fine-tunes the grid element shape measure. The element internal shape function that is optimized is the functional defined in Vasilevskii and Lipnikov [24]. Pragmatic was started as a hybrid threads and MPI parallel code. Since then, the enthusiasm for hybrid parallelism has waned on the solver side, so a purely distributed memory approach was favored in Pragmatic. CAD support functionalities have been added since the publication of Ibanez et al. [13], and geometry projection is handled by EGADS.

\section{E. Feflo.a}

Feflo.a is an adaptation code developed at INRIA. It is based on a two-step procedure to output a unit grid [25, 26]. The first step aims at improving the edge length distribution with respect to the input metric field. In its original version, only classical edge-based operators (insertion and collapse) are used during this step. The second step is optimization of the grid element shape measures with node smoothing and tetrahedra edge and face swaps. Feflo.a can handle nonmanifold surface and/or volume grids composed of simplicial elements. For the surface grid adaptation, a dedicated surface metric is used to control the deviation of the metric and surface curvature. This surface metric is then combined with the input metric. New points created on the surface are projected to a (fine) background surface grid and optionally CAD via the EGADS API.

Recently, classical edge-based operators have been replaced by a unique cavity-based operator [27, 28]. This cavity-based operator simplifies code maintenance, increases the success rate of grid modifications, has a constant execution time for many different local operations, and robustly inserts boundary layer grids [29]. When the cavity operator is combined with advancing-point techniques, it outputs metric-aligned and metric-orthogonal grids [30].

\section{Integrated Grid Adaptation Processes}

The grid adaptation process involves a flow (and adjoint) solver, error estimation, metric calculation, and grid mechanics. The target size for the adapted grid is specified in the form of a $3 \times 3$ symmetric metric tensor at each node of the grid. The metric defines an ellipsoid where the eigenvectors of the metric represent the direction of principle axes and the eigenvalues of the metric represent the length of these axes. The metric specifies the desired anisotropic grid density. The complexity of the metric is computed and the metric is globally scaled to produce an adapted grid with a target number of nodes and elements. Common file formats for grids, solutions, and metric fields were used so that components of these integrated adaptive processes could be evaluated independently.

\section{A. GGNS}

GGNS (General Geometry Navier-Stokes) is a Boeing-developed flow solver built upon the Streamline Upwind/Petrov Galerkin (SUPG) stabilized finite element (FE) discretization. The code uses piecewise linear finite elements resulting in a second order accurate discretization. Additional first-order artificial viscosity built upon the nodal DG(0) discretization is added for shock capturing. The indicator triggering this additional stabilization is based on the oscillation of the Mach number across a cell. The solver can work with unstructured grids of mixed-element type (tetrahedrons, prisms, and pyramids) as well as pure tetrahedral grids. The number of degrees of freedom for the second-order SUPG scheme is equal to the number of nodes in the computational grid. The discretization is "node-based" in the sense that it is conservative over the dual volumes of an unstructured grid. More details on discretization used in the GGNS solver, including the particular choices of discretization variables and special treatment of the essential boundary conditions via the Lagrange-multiplier based technique [31], can be found in Kamenetskiy et al. [32].

The discrete nonlinear solver in the GGNS code implements a variant of the Newton-Krylov-Schwartz algorithm. On the code level, this is accomplished using the PETSc solver framework [22]. Time stepping is employed to drive to the steady state solution. On each time step, an exact Jacobian matrix for the discretization is formed by an automatic differentiation technique. The linear system arising from the Newton's method is approximately solved using GMRES 
with a drop-tolerance-based BILUT preconditioner (locally on subdomains) implemented in the context of the additive Schwartz method with minimal overlap [33]. Right preconditioning is employed to maintain consistency between the nonlinear and linear residuals. The compact stencil property of the SUPG scheme helps to reduce the fill-in levels in the approximate factorization, thereby reducing the memory footprint.

A line search is applied along the direction provided by the approximate solution of the linear system. Residual decrease and physical realizability of the updated state are tracked during the line search. A heuristic feedback algorithm is implemented to communicate failure of the line search back to the time-stepping algorithm, so that the CFL number can be increased or decreased as necessary. There is no upper preset limit for the CFL number in the time-marching algorithm; so Newton-type quadratic convergence (or, at least, superlinear, due to inexact linear solves) is routinely achieved at steady state.

The adaptive grid process consists of a sequence of adaptation cycles. Each adaptation cycle consists of running a flow solution to convergence, generating a sizing request for the next grid, generating a new adaptive grid that conforms to the sizing request, and interpolating the solution to the new grid. The sequence of adaptation cycles is continued until the output of interest reaches convergence.

The target sizing request is typically computed from an error estimate derived from the CFD flow solution. Error indicators generally fall into two classes, feature-based methods that are derived from properties of the flow field such as the Mach Hessian, or output-based methods that are derived from minimization of the errors associated with a functional output such as integrated lift or drag. The Mach Hessian for each cell/element is computed from the flow solution. EPIC converts the Hessians to adaptation metrics via a cell-centered modification of the goal-oriented error estimate of Alauzet and Loseille [14], which minimizes the $L_{p}$ norm of interpolation error of the scalar field for a given grid complexity. A continuous metric field is generated by Log-Euclidean interpolation of the metrics to the grid nodes.

\section{B. Wolf}

Wolf solves the RANS system with the SA turbulence model. The spatial discretization of the fluid equations is based on a vertex-centered, finite-element/finite-volume formulation on unstructured grids. It combines an HLLC [34] upwind scheme for computing the convective fluxes and the Galerkin centered method for evaluating the viscous terms. Second-order space accuracy is achieved through a piecewise linear interpolation based on the Monotonic Upwind Scheme for Conservation Law (MUSCL) procedure, which uses a particular edge-based formulation with upwind elements. A specific slope limiter is employed to damp or eliminate spurious oscillations that may occur in the vicinity of discontinuities.

The linearization for Newton's method can use either the Lower-Upper Symmetric Gauss-Seidel (LU-SGS) approximate factorization or the Symmetric Gauss-Seidel (SGS) relaxation. The LU-SGS and SGS are very attractive because they use an edge-based data structure that can be efficiently parallelized. The GMRES method can use either of these methods as a preconditioner.

Drag error and Mach interpolation error is controlled with the metrics described by Alauzet and Loseille [14], Drag error is controlled through an adjoint-gradient-weighted Hessian of the continuous fluxes. Mach interpolation error is minimized in the $L_{p}$ norm of interpolation error of the scalar field for a given grid complexity. Feflo.a modifies the grid to conform to the solution-adaptive metric.

\section{FUN3D-FV}

FUN3D-FV [35, 36] is a finite-volume Navier-Stokes solver in which the flow variables are stored at the vertices or nodes of the grid. FUN3D-FV solves the equations on mixed-element grids, including tetrahedra, pyramids, prisms and hexahedra. At interfaces between neighboring control volumes, the inviscid fluxes are computed using the Roe [37] approximate Riemann solver based on the values on either side of the interface. For second-order accuracy, interface values are extrapolated from the vertices with gradients computed at the grid vertices. These gradients are reconstructed with an unweighted least-squares technique [35].

The full viscous fluxes are discretized using a finite-volume formulation in which the required velocity gradients on the dual faces are computed using the Green-Gauss theorem. On tetrahedral grids this is equivalent to a Galerkin type approximation. The solution at each time step is updated with a backwards Euler time-integration scheme. At each time step, the linear system of equations is approximately solved with a multicolor point-implicit procedure [38]. Local time-step scaling is employed to accelerate convergence to steady state. The SA turbulence model is loosely-coupled to the meanflow equations, where the meanflow and turbulence model equations are relaxed in an alternating sequence.

The SA turbulence model requires the distance from every node to the nearest noslip boundary condition. The 
standard wall distance calculation in FUN3D-FV finds the nearest surface node and then searches adjacent triangles to see if they are closer than the closest surface node. The standard wall distance method is inaccurate if the closest triangle is not adjacent to the closest surface node. To provide an accurate wall distance, which is critical to the SA model, an alternative method is used on adapted grids. The alternative method encloses each surface triangle in a bounding box. These bounding boxes are stored in an Alternating Digital Tree (ADT) [39] for fast searches. The alternative wall distance method finds the closest surface triangle for adapted unstructured grids. The impact of the standard and alternative wall distance implementations will be shown for the adaptive grid cases.

The metric is formulated to control the $L_{p}$ norm of the interpolation error of a solution scalar field. To form the metric, a Hessian of the scalar field is reconstructed by recursive application of a gradient reconstruction scheme. The gradient is computed in each element and a volume-weighted average is collected at each vertex [14]. The second-derivative Hessian terms are formed by computing the reconstructed gradients of these gradients formed in the first pass. The Hessian is then decomposed into eigenvalues and eigenvectors. The metric is formed by recombining the absolute value of the eigenvalues with the eigenvectors to ensure the metric is symmetric positive definite. The metric at each vertex is scaled to control the $L_{p}$ norm [14]. The graduation of the metric field is limited to 1.5 isotropically in the metric space [40]. The complexity is computed, and the metric is globally scaled to set its complexity to a specified value. The grid is adapted by refine to conform to the metric.

\section{Hemisphere Cylinder Adaptation}

The Hemisphere Cylinder geometry is based on a wind tunnel study by Hsieh [41, 42]. For this study, the outer domain boundary is described as a hemisphere, where the exit plane intersects the cylinder (see Fig. 11). The Hemisphere Cylinder was previously used for RANS solver verification with a different outer boundary shape and grid topology [6]. The zero angle of attack case is the focus here to compare multiple flow solvers on uniformly-refined grids to $L_{p}$ Mach grids adapted with FUN3D-FV and refine.

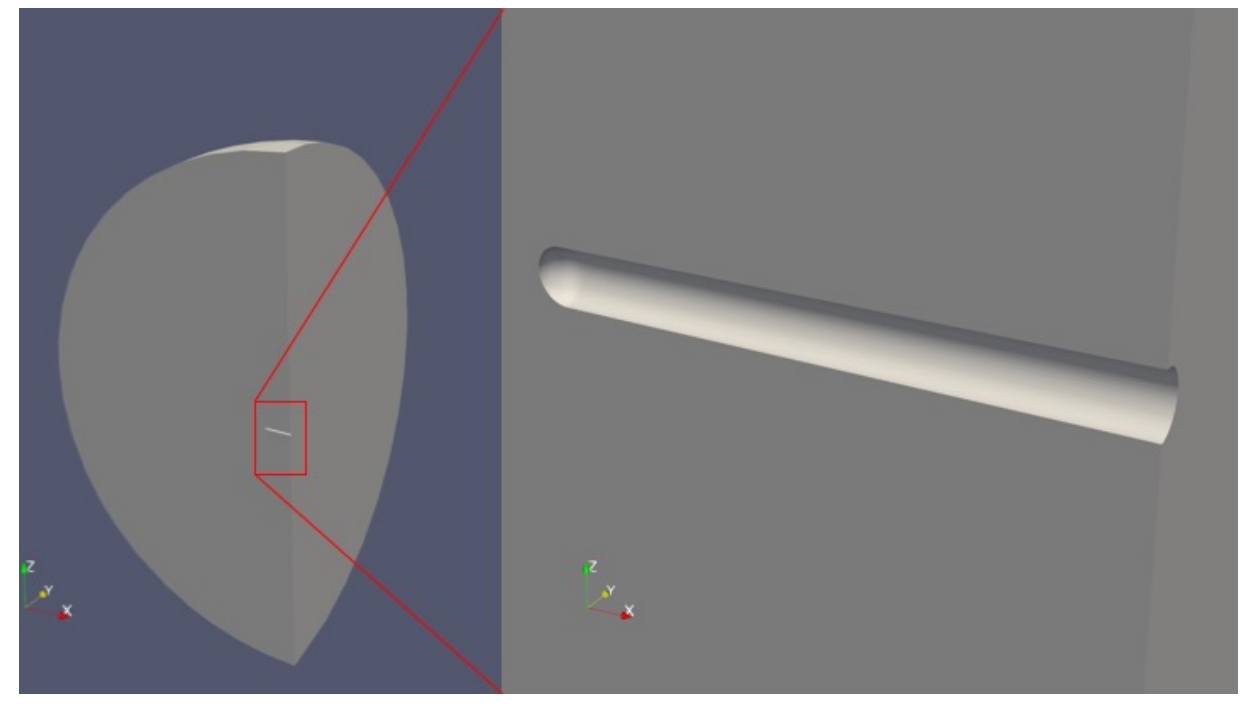

Fig. 1 Illustration of the Hemisphere Cylinder geometry.

Three Mach $L_{p}$ strategies are explored for the Hemisphere Cylinder in Fig. 2 Uniformly-refined, fixed grid results obtained from the TMR are also presented with dashed lines to indicate the expected drag component values for well-resolved grids. A characteristic grid size $h$ is estimated as the inverse of the cube root of the number of degrees of freedom in the grid (nodes for vertex-based methods and cells for element-based methods). These adapted grids are unstructured tetrahedra with no imposed structure near the wall. All surface nodes constrained by geometry edges or faces have been evaluated with EGADS. The continuous refinement trajectory (Cont. Ref.) increases the specified metric complexity after each flow solution. The continuous refinement trajectory uses the standard FUN3D-FV wall distance method (Stand. Dist.). The Step refinement trajectories hold complexity constant for five grid adaptation cycles before increasing it, which produces multiple drag values at a given grid size. The order of the $L_{p}$ norm is indicated in the figure legend. The Step refinement trajectories use the alternative wall distance method. The Cont. Ref. and 
Step trajectories converge faster to the fine, fixed-grid pressure drag than FUN3D-FV on the fixed grids. The Step refinement with the alternative distance function is much closer to the fine, fixed-grid viscous drag than Cont. Ref. with the standard distance function. The Cont. Ref. is omitted from a zoom view of the viscous drag in Fig. 2(c), where the Step trajectories are approximately two counts higher in drag. Example grids from the Cont. Ref. trajectory are used in the Metric Conformity section to evaluate adaptive grid mechanics. While the Cont. Ref. trajectory is slower to converge to the fine, fixed-grid drag, the grid metric distribution is representative of either case.

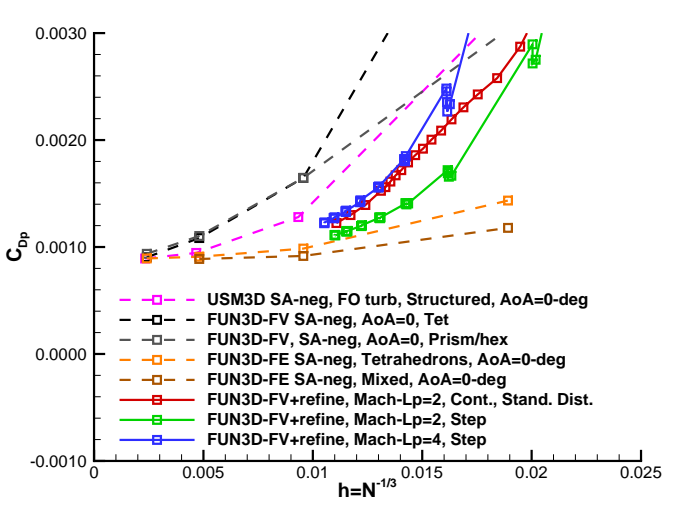

(a) Pressure component of drag coefficient.

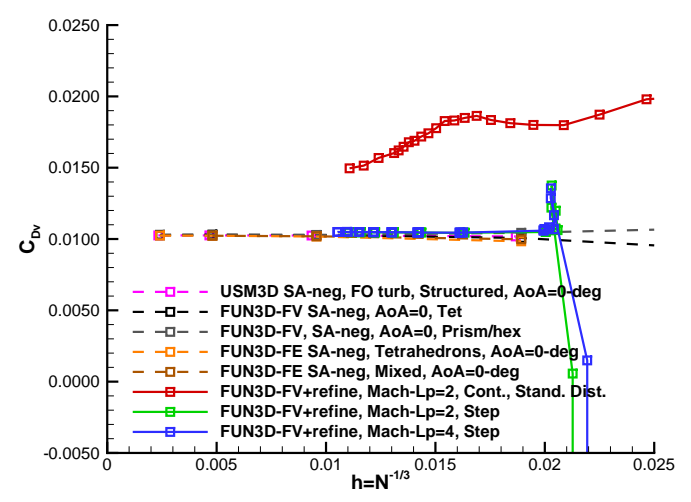

(b) Viscous component of drag coefficient.

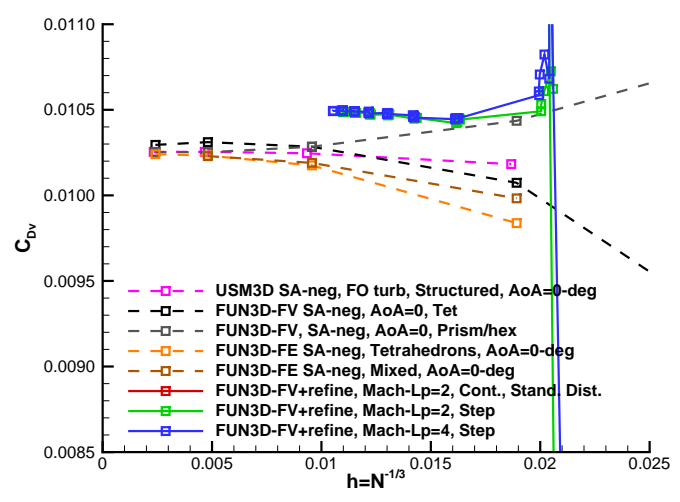

(c) Viscous component of drag coefficient, zoom.

\section{Fig. 2 Hemisphere Cylinder FUN3D-FV+refine $L_{p}$ Mach adaptation at a series of fixed complexities.}

A possible explanation for the high viscous drag prediction in the Cont. Ref. trajectory of Fig. 2 2 is a violation of an assumption in the distance to the wall calculation that is critical for the SA turbulence model. The computed wall distance along the symmetry plane one diameter from the nose is shown in Fig. 3 . The expected result is parallel lines, but the wall distance is larger than the expected distance for some nodes. The anisotropic unstructured grid violates an assumption in the standard FUN3D-FV wall distance calculation, where the closest surface triangle to an off-body node is expected to be adjacent to the closest surface node to an off-body node.

The coefficient of pressure and skin friction on the final grid for the Step Ref. trajectories is examined in Fig. 4. Differences in skin friction are larger between $L_{p=2}$ and $L_{p=4}$ norms than coefficient of pressure. Skin friction on the nose is more concentric for $L_{p=4}$. The final surface grids are shown in Fig. 5. Both of these grids have the same requested metric complexity and approximately the same number of nodes. The $L_{p=4}$ metric requests more refinement tangential to the surface. The symmetry plane grid indicates normal refinement initially at the model and in the region of high Mach curvature off the model. 


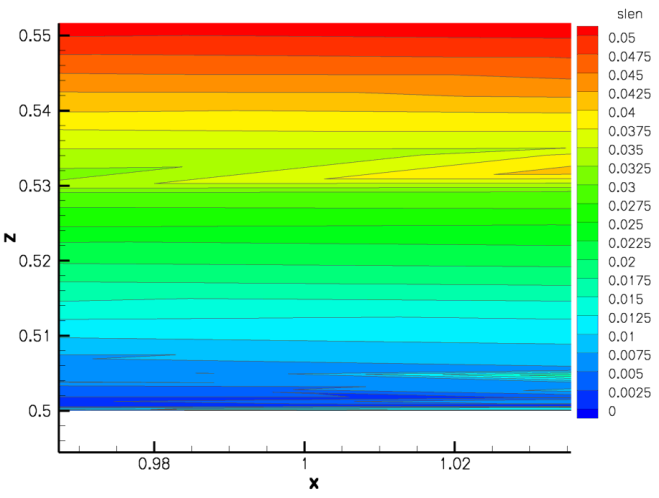

Fig. 3 Hemisphere Cylinder FUN3D-FV+refine $L_{p=2}$ Mach adaptation, final grid, distance to solid wall.

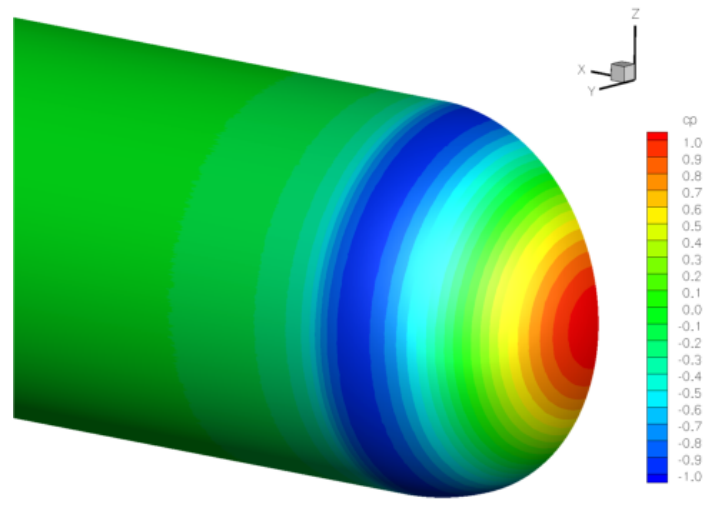

(a) $L_{p=2}$ Pressure coefficient.

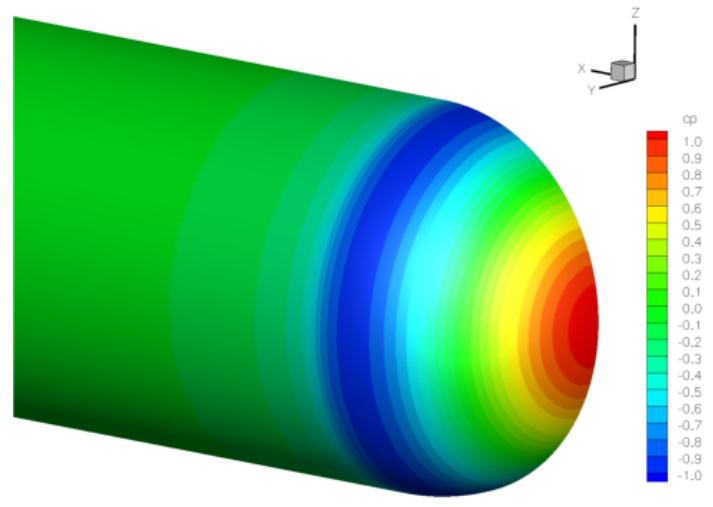

(c) $L_{p=4}$ Pressure coefficient.

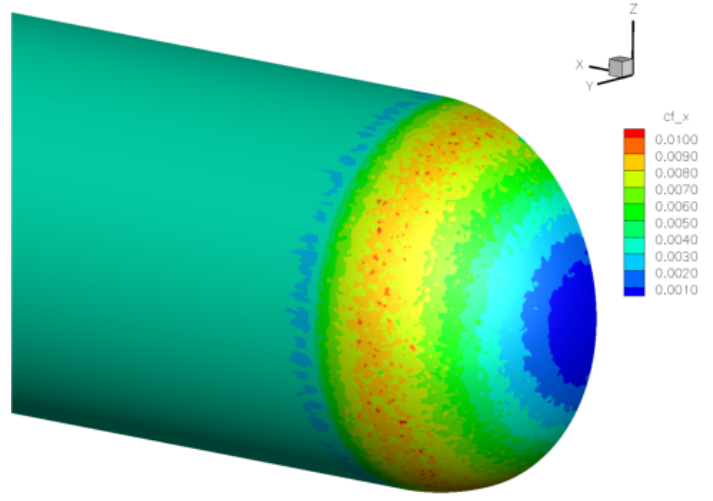

(b) $L_{p=2}$ Skin friction $x$-component.

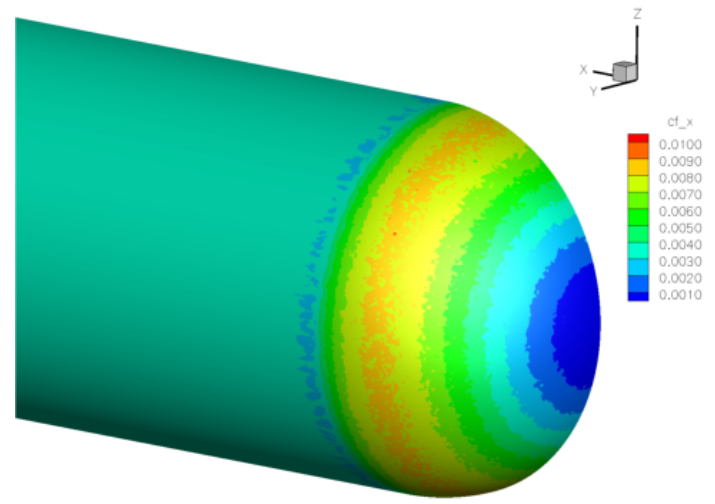

(d) $L_{p=4}$ Skin friction $x$-component.

Fig. 4 Hemisphere Cylinder FUN3D-FV+refine $L_{p}$ Mach adaptation, final grid solution. 


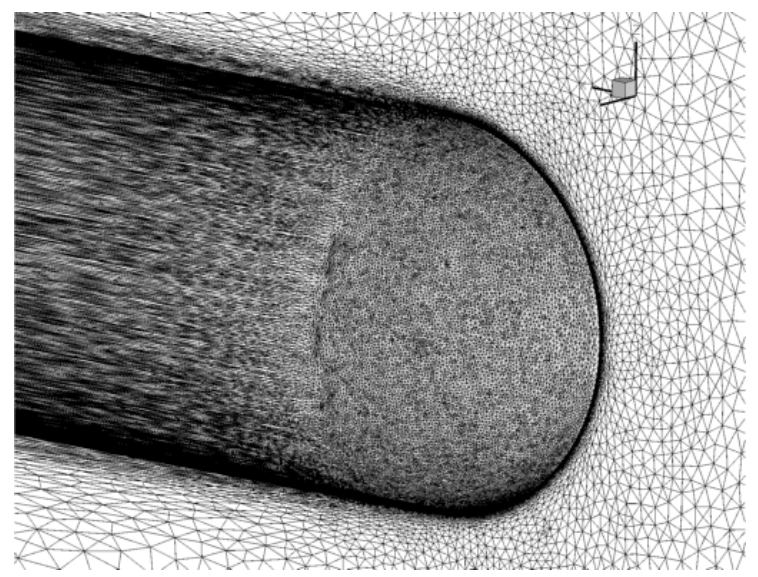

(a) $L_{p=2}$ final grid on nose and symmetry plane.

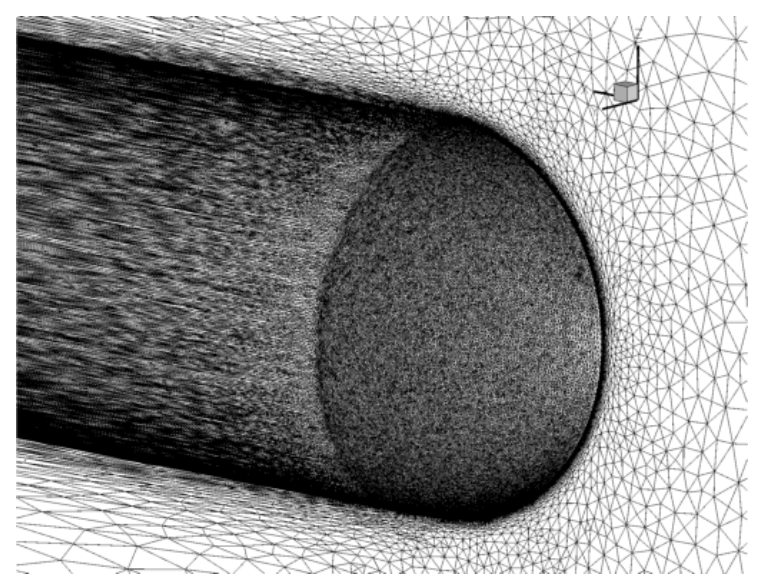

(c) $L_{p=4}$ final grid on nose and symmetry plane.

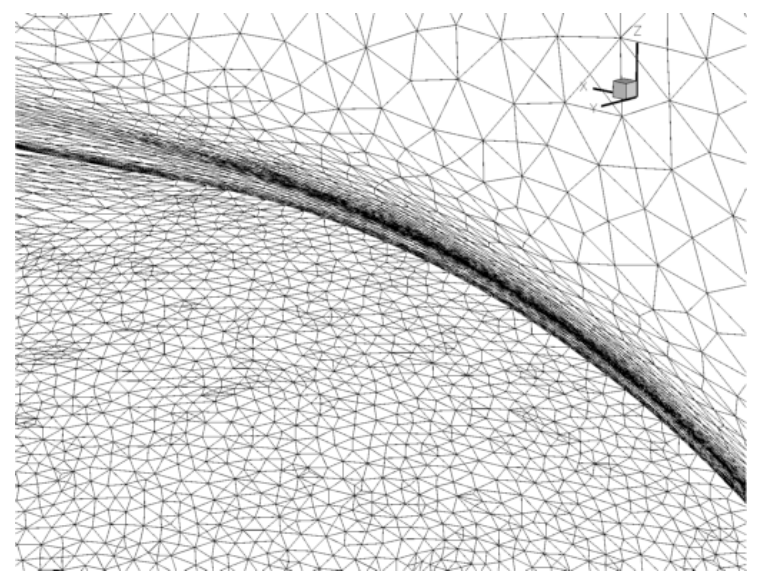

(b) $L_{p=2}$ final grid on shoulder and symmetry plane.

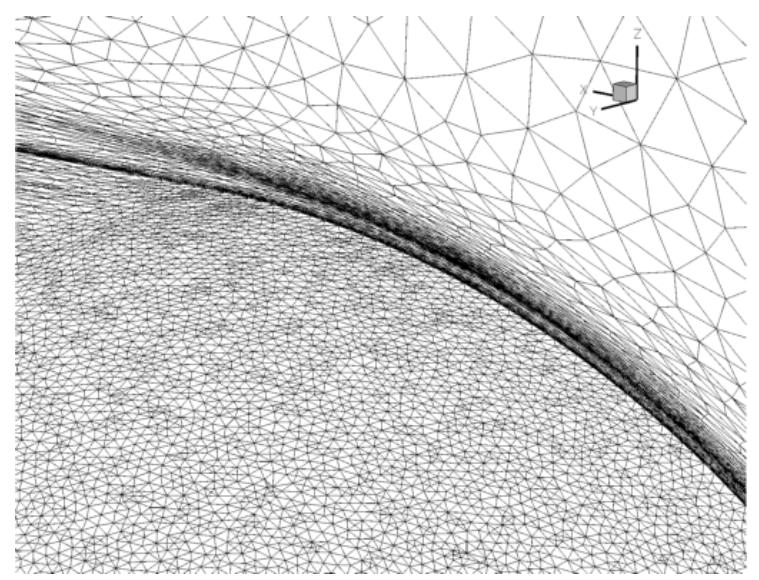

(d) $L_{p=4}$ final grid on shoulder and symmetry plane.

Fig. 5 Hemisphere Cylinder FUN3D-FV+refine $L_{p}$ Mach adaptation, final grid. 


\section{Alternate Hemisphere Cylinder Geometry}

The original Hemisphere Cylinder domain has adjacent boundary faces with boundary conditions that are not compatible for some flow solvers. To address this concern, the Alternate Hemisphere Cylinder Geometry was proposed with a spherical outer boundary. The model is completed by appending a body described by Groves, Huang, and Chang [43] to the cylinder. This avoids the intersection of the model with an exit plane. For verification exercises, the original and alternative geometries should be studied separately. Uniformly-refined, fixed grids are also available for this case. Pressure and viscous components of GGNS adaptive grid drag calculations are compared to GGNS on these fixed grids in Fig. 6 for low angles of attack and Fig. 7 for higher angles of attack. Only node insertion, edge collapse, and element swaps are used for EPIC-ICS to reduce execution time. Mach $L_{p=4}$ and drag error adaptation converge faster to the uniformly-refined drag value. Lift error and Mach $L_{p=2}$ converge slower on coarse grids. On grids with $h$ smaller than 0.012 , the adaptive schemes produce similar trajectories at this scale.

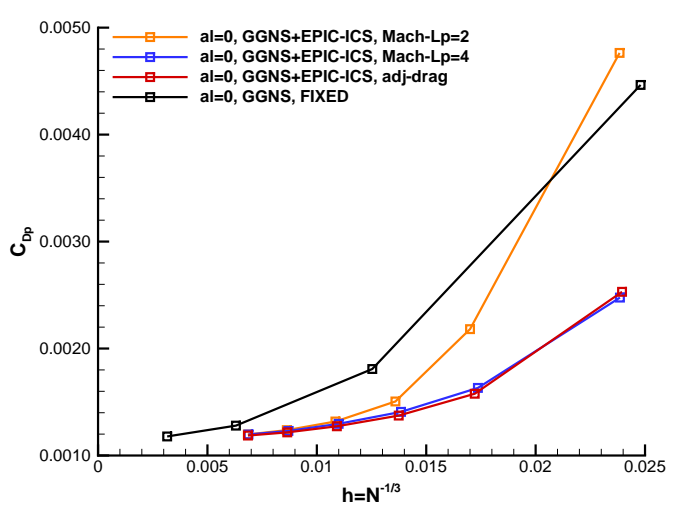

(a) Pressure component of drag coefficient, $\alpha=0$.

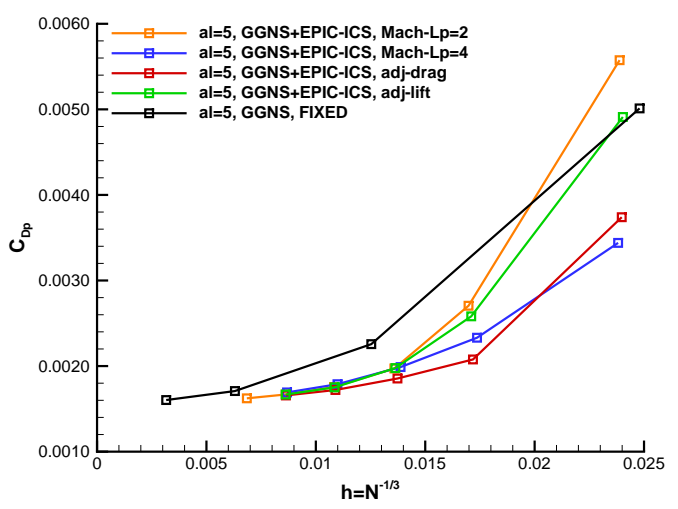

(c) Pressure component of drag coefficient, $\alpha=5$.

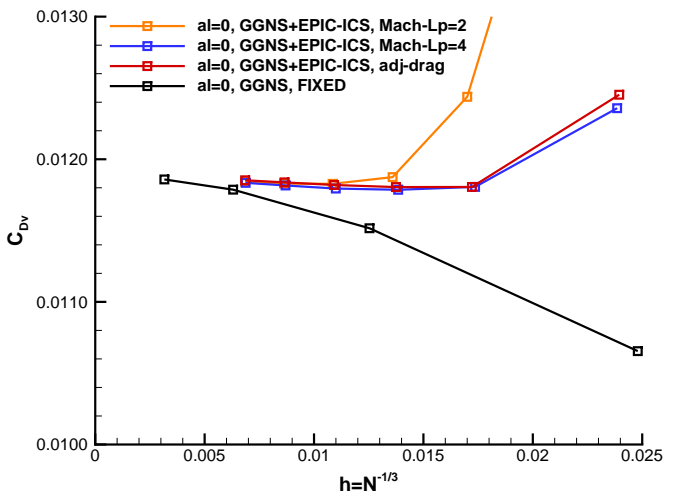

(b) Viscous component of drag coefficient, $\alpha=0$.

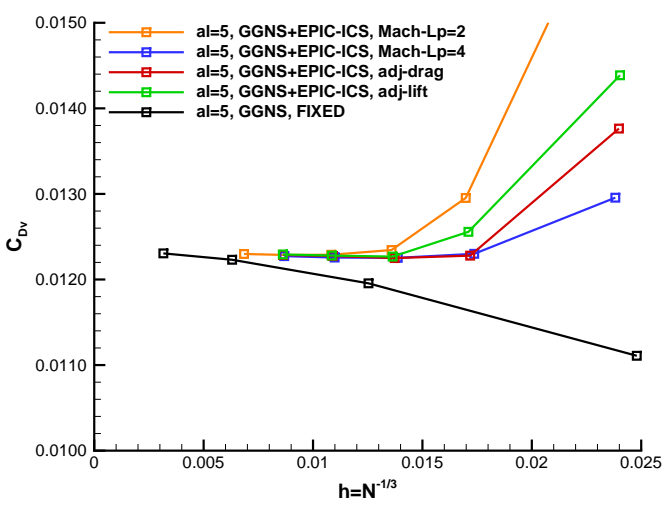

(d) Viscous component of drag coefficient, $\alpha=5$.

Fig. 6 Alternate Hemisphere Cylinder GGNS+EPIC-ICS adaptation, low angles of attack. 


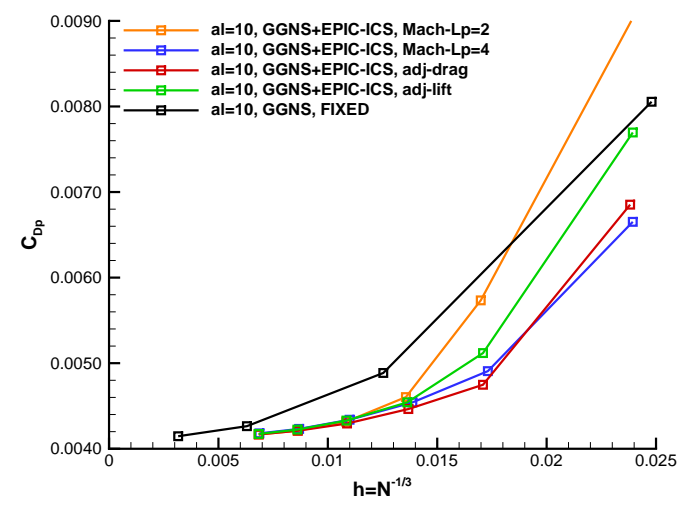

(a) Pressure component of drag coefficient, $\alpha=10$.

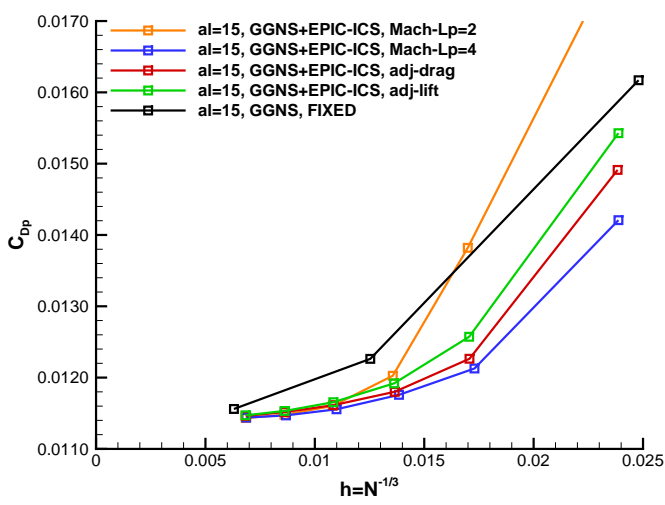

(c) Pressure component of drag coefficient, $\alpha=15$.

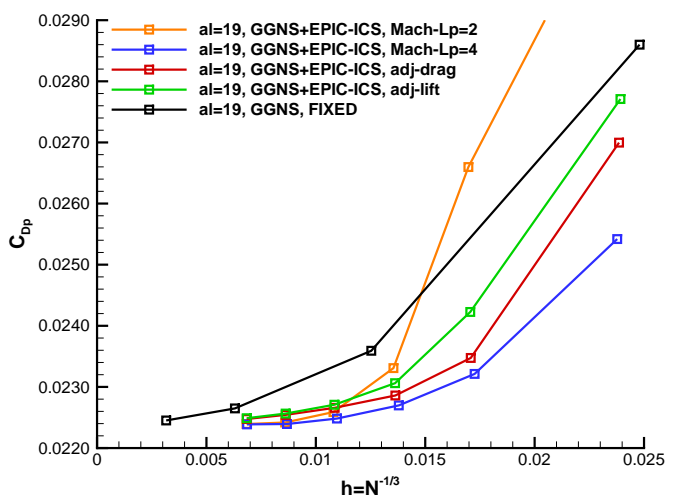

(e) Pressure component of drag coefficient, $\alpha=19$.

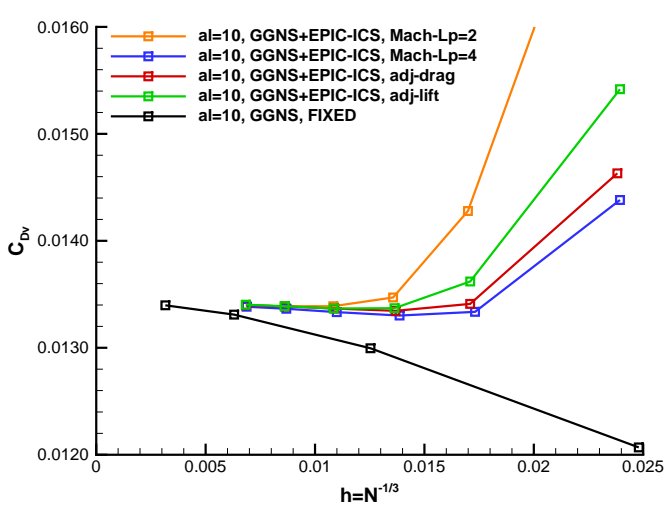

(b) Viscous component of drag coefficient, $\alpha=10$.

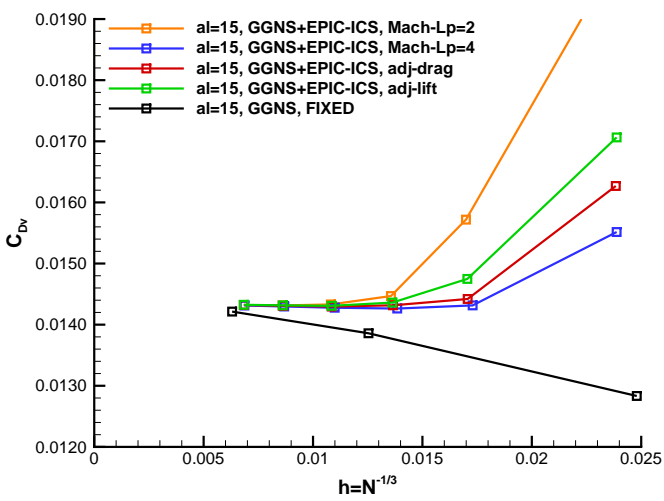

(d) Viscous component of drag coefficient, $\alpha=15$.

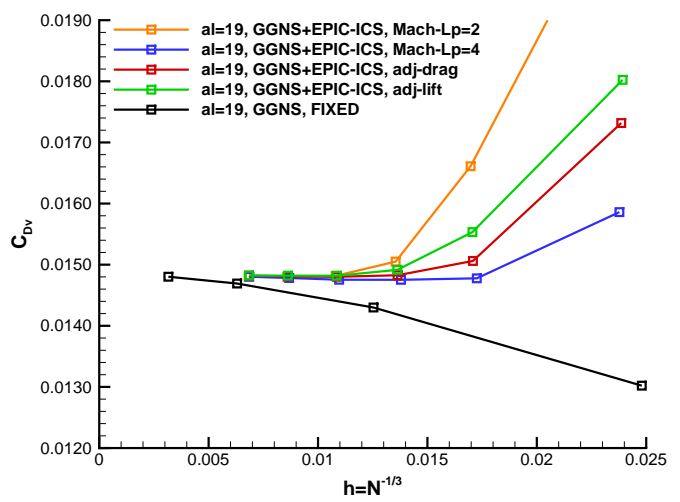

(f) Viscous component of drag coefficient, $\alpha=19$.

Fig. 7 Alternate Hemisphere Cylinder GGNS+EPIC-ICS adaptation, high angles of attack. 


\section{ONERA M6 Adaptation}

The ONERA M6 wing was originally described in an AGARD report [44]. The geometry has been modified from this original description to have a sharp training edge and a well-defined wing tip shape by Mayeur et al. [45, 46]. Uniformly-refined, fixed-grid results obtained from the TMR indicate the expected force and moment values for wellresolved grids. The pressure and viscous components of drag converge more consistently on these uniformly-refined grids than lift or pitching moment. Adaptive GGNS results are shown as solid lines in Fig. 8 with the TMR results as dashed lines. Adaptive FUN3D-FV results are shown as solid lines in Fig. 9. FUN3D-FV solutions are also computed on GGNS lift-adapted grids from Fig. 8. The vertical scale is smaller for GGNS plots because the difference between GGNS adaptive results and the uniformly-refined grids are smaller. Adaptive Wolf results are shown as solid lines in Fig. 10. Wolf solutions are also computed on GGNS lift adapted grids from Fig. 8 .

The GGNS adaptive trajectories increase complexity in a number of steps. Only node insertion, edge collapse, and element swaps are used for EPIC-ICS to reduce execution time. The drag components monotonically converge at constant complexity. The lift and pitching moment show jitter during the constant complexity phases. The GGNS+EPICICS forces and moment converge rapidly. Mach $L_{p=2}$ is slower to converge as compared to the other adaptive schemes, which appear to have a lower error than the uniformly-refined, fixed-grids for grids finer than $h=0.018$.

The Cont. Ref. FUN3D-FV adaptation trajectory increases complexity each time the metric is computed. The continuous refinement trajectory uses the standard FUN3D-FV wall distance method (Stand. Dist.). The Step trajectories hold complexity constant for 5 adaptations before an increase in complexity. The Step refinement trajectories use the alternative wall distance method. The FUN3D-FV Step trajectories with the alternative wall distance method appear to converge toward the uniformly-refined, fixed-grid values. Adaptive grid lift and pitching moment convergence to the uniformly-refined, fixed-grid values appears to be stalled for Cont. Ref. FUN3D-FV with the standard wall distance method. The FUN3D-FV solutions on the Lift adapted GGNS+EPIC-ICS use the alternative wall distance method are near the fixed-grid FUN3D-FE. Example grids from the Cont. Ref. trajectory are used in the Metric Conformity section to evaluate adaptive grid mechanics. While the Cont. Ref. trajectory is slower to converge to the fine, fixed-grid forces and moment, the grid metric distribution is representative of the other trajectories.

Adaptive Wolf trajectories show monotonic convergence toward the uniformly-refined, fixed-grids. Only the finial grid in a series of fixed-complexity adaptation steps is shown. The drag-error-based metric converges slower than Mach $L_{p}$ for these forces and moment. The Wolf solutions on the Lift adapted GGNS+EPIC-ICS are near the uniformly-refined, fixed grids, but there is more variation with grid refinement than with GGNS or FUN3D-FV.

The wall distance calculation assumption violation discovered with the FUN3D-FV Hemisphere Cylinder adaptation case is also present on the adapted FUN3D-FV ONERA M6 cases. The standard method for computing distance to the wall on the final grid of the $L_{p=2}$ Cont. Ref. adaptation is shown for the symmetry plane near the trailing edge in Fig. 11 Equidistant contours are expected, but there are nodes with larger wall distance than expected. The anisotropic unstructured grid violates an assumption in the standard FUN3D-FV wall distance calculation, where the closest surface triangle to an off-body node is expected to be adjacent to the closest surface node to an off-body node. Errors in wall distance negatively impact the SA turbulence model accuracy.

To understand the impact of wall distance calculation, the FUN3D-FV solution is provided for a GGNS+EPIC-ICS grid with the alternative and standard wall distance methods. The forces and moment are shown in Table 1 , where FUN3D-FV with the alternative wall distance method is closer to GGNS than FUN3D-FV with the standard wall distance method. The GGNS+EPIC-ICS Mach- $L_{p=4}$ grid has $154 \mathrm{~K}$ nodes $(h=0.0186)$. The tetrahedral grid intersected at two constant span locations is shown in Fig. 12. The grid shows clustering at the leading edge, shock, wake, and boundary layer. These adapted grids lack the orthogonality that is typically observed in fixed grids. The extremely unstructured nature of these grids violates the assumptions of the standard FUN3D-FV wall distance method.

Mach number at two constant span locations is shown in Fig. 13. The FUN3D-FV solution with the alternative wall distance method appears similar to GGNS with small differences near shocks. The FUN3D-FV solutions with the standard wall distance method indicate a thicker boundary layer in the aft portion of the wing and the wake. The upper surface shock is more diffuse and the supersonic regions are smaller than the other methods on the same grid. The only difference between these two FUN3D-FV simulations is the wall distance method. 
Table 1 Forces and Moment for GGNS+EPIC-ICS Mach- $L_{p=4}$ grid with 154K nodes.

\begin{tabular}{lrrrr} 
Code & $C_{D p}$ & $C_{D v}$ & $C_{L}$ & $C_{M y}$ \\
\hline GGNS & 0.01223 & 0.00549 & 0.27196 & -0.19355 \\
FUN3D-FV, alternative wall distance & 0.01250 & 0.00526 & 0.26931 & -0.19143 \\
FUN3D-FV, standard wall distance & 0.01714 & 0.01001 & 0.24643 & -0.17216
\end{tabular}

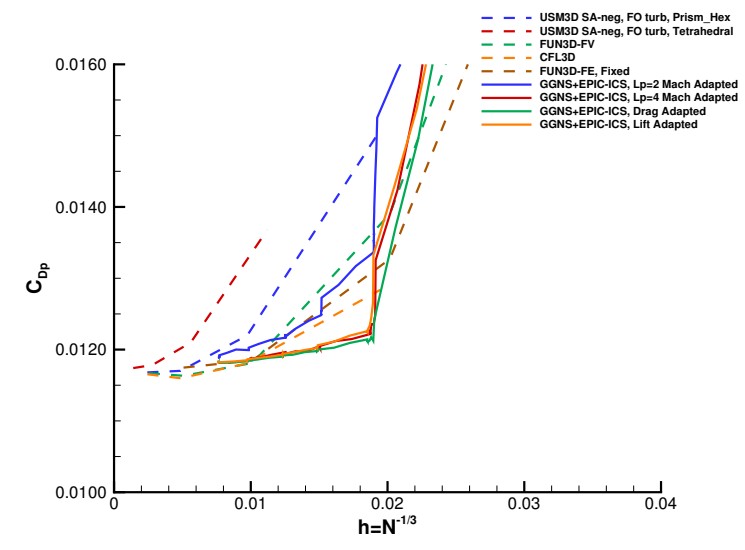

(a) Pressure component of drag coefficient.

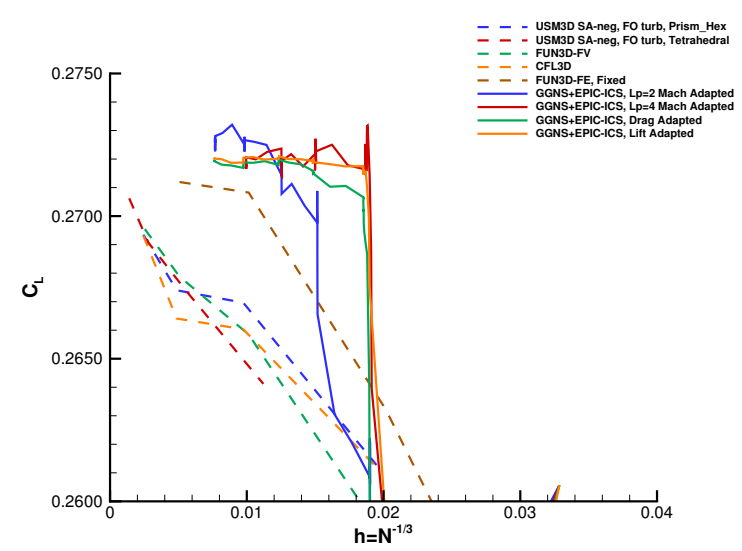

(c) Lift coefficient.

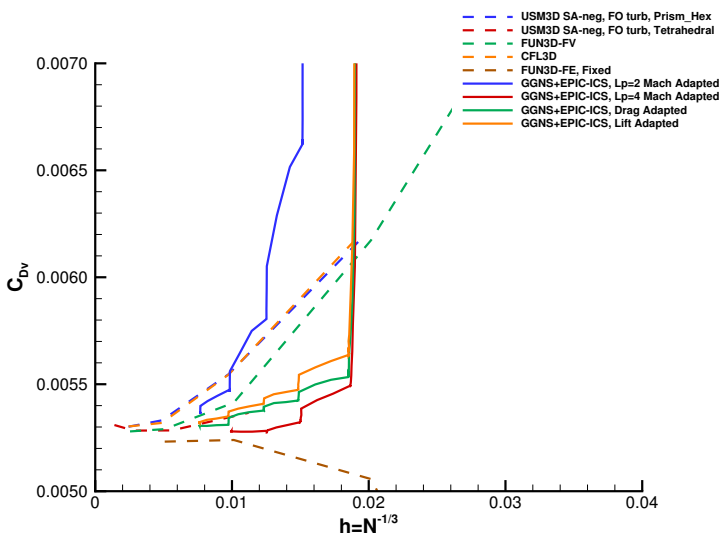

(b) Viscous component of drag coefficient.

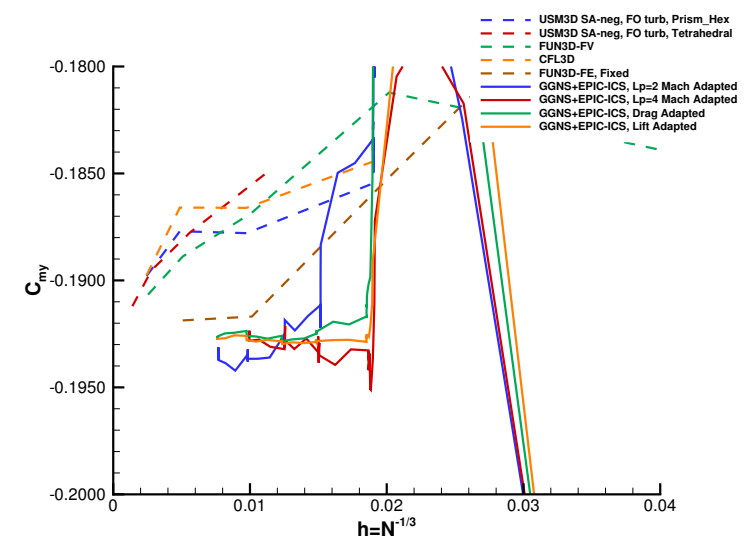

(d) Pitching moment coefficient.

Fig. 8 GGNS ONERA M6 force and moment convergence. 


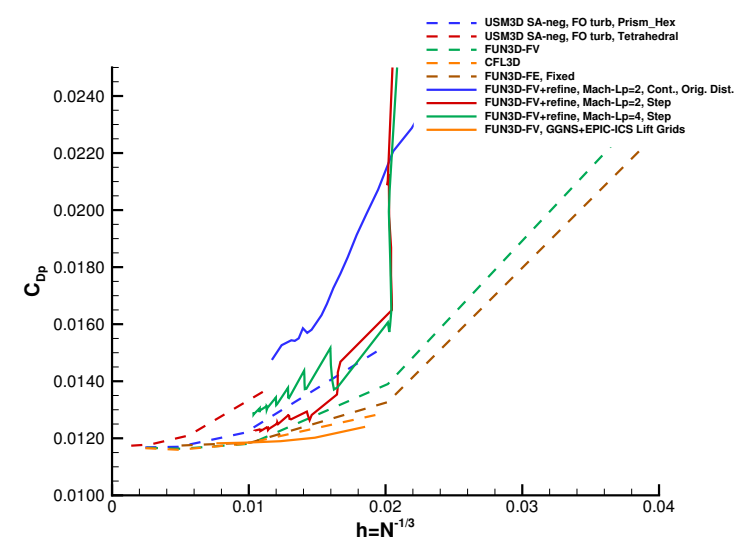

(a) Pressure component of drag coefficient.

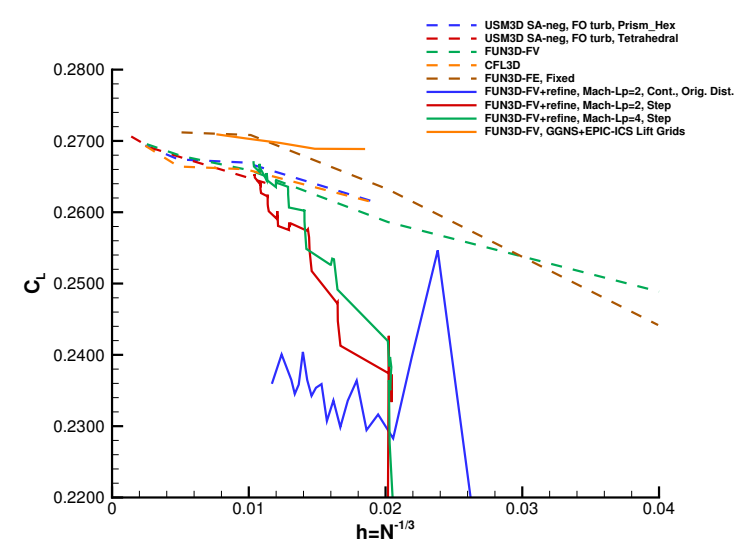

(c) Lift coefficient.

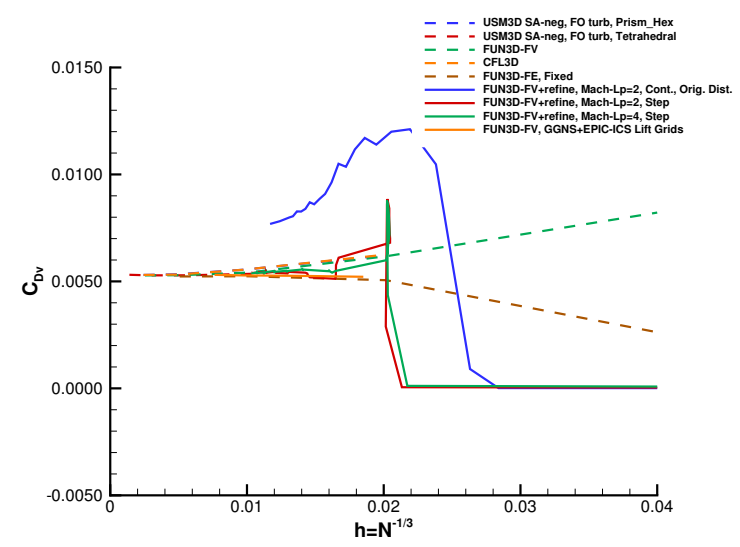

(b) Viscous component of drag coefficient.

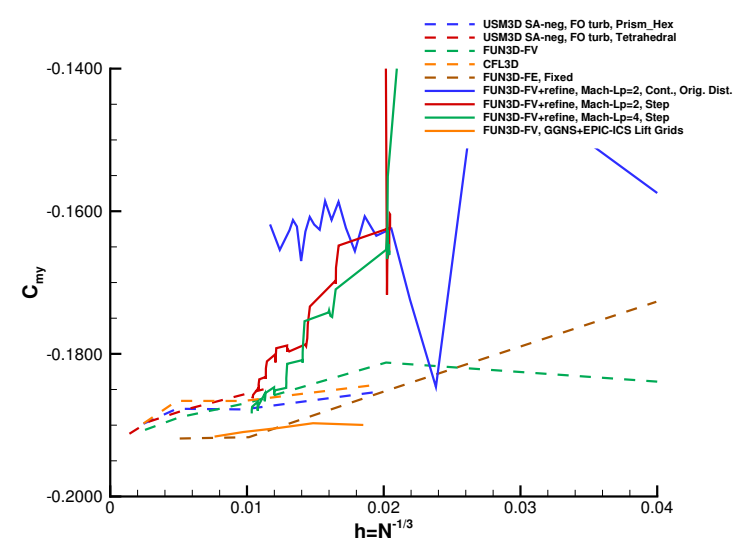

(d) Pitching moment coefficient.

Fig. 9 FUN3D-FV ONERA M6 force and moment convergence. 


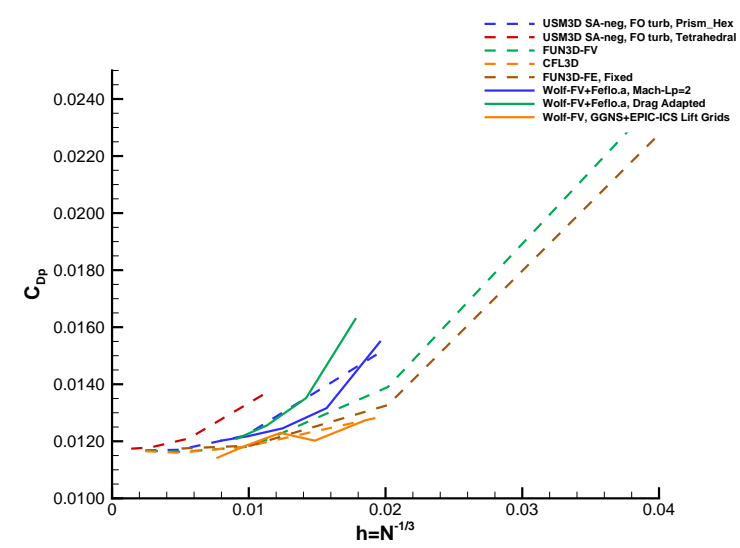

(a) Pressure component of drag coefficient.

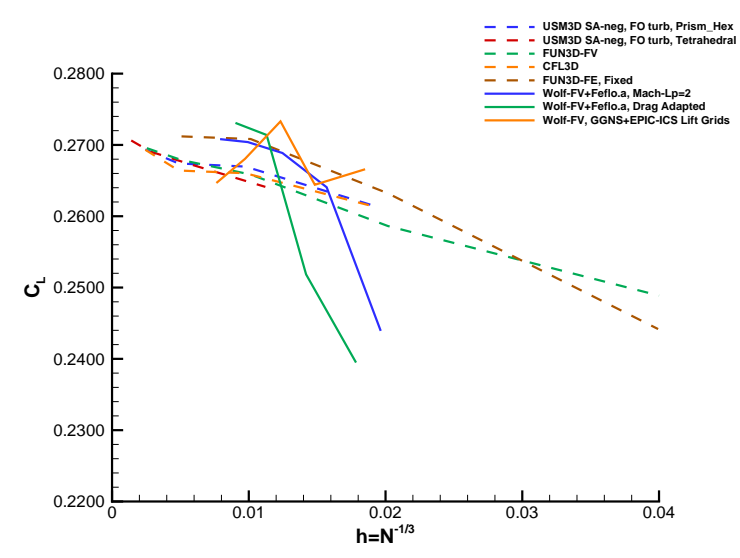

(c) Lift coefficient.

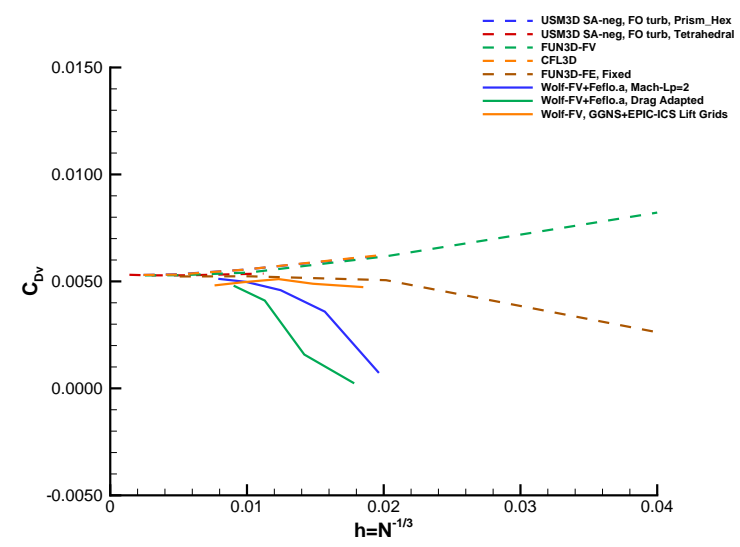

(b) Viscous component of drag coefficient.

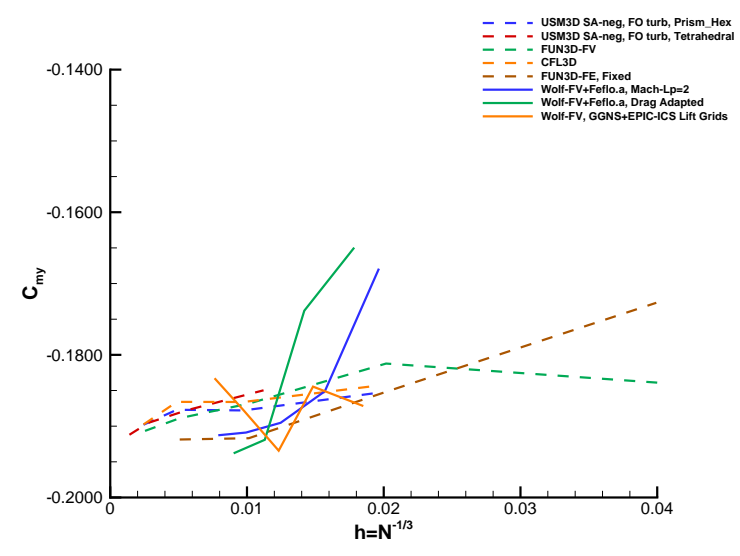

(d) Pitching moment coefficient.

\section{Fig. 10 Wolf ONERA M6 force and moment convergence.}




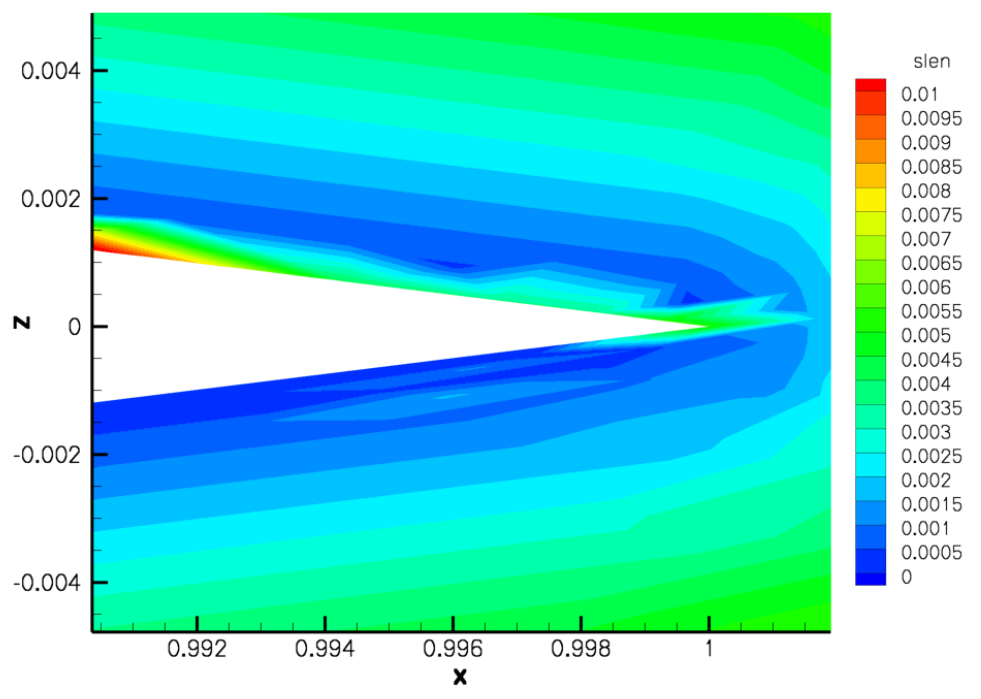

Fig. 11 ONERA M6 FUN3D-FV+refine $L_{p=2}$ Mach adaptation, final grid, standard method to compute distance to solid wall at trailing edge.

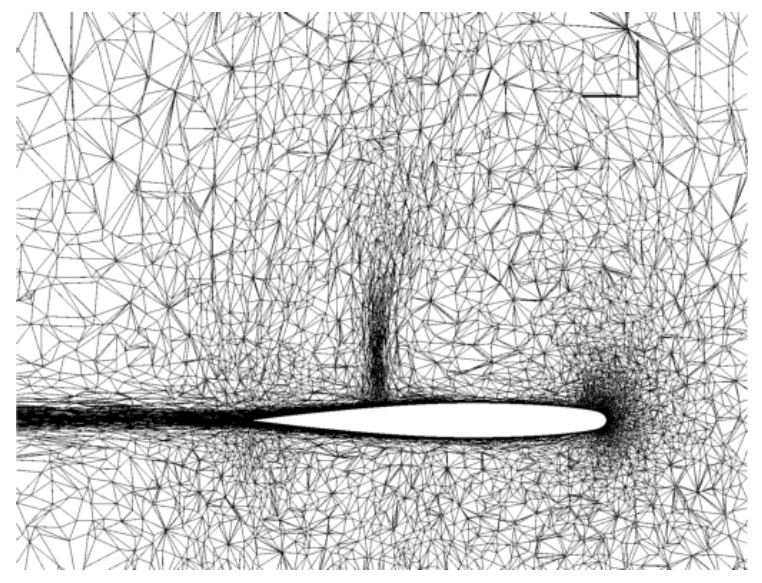

(a) GGNS+EPIC-ICS, $y / b=0.2$.

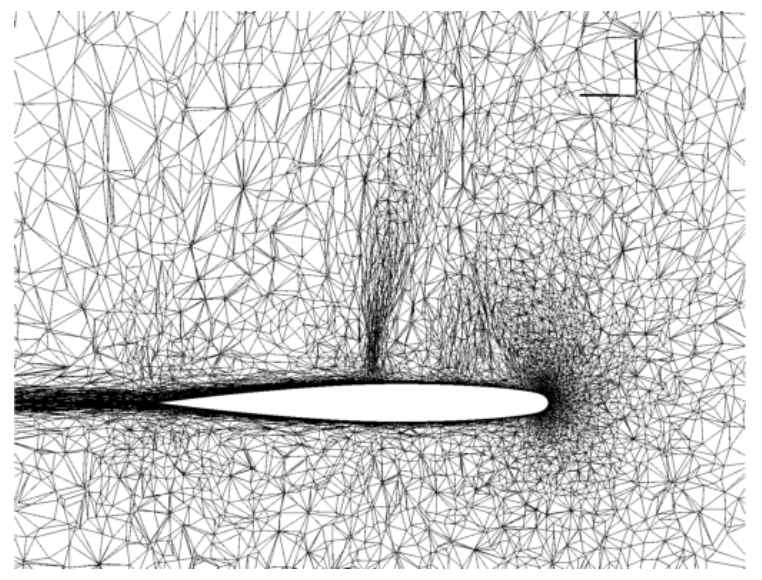

(b) GGNS+EPIC-ICS, $y / b=0.7$.

Fig. 12 Constant span slices of the iteration 17 grid of GGNS+EPIC-ICS $L_{p=4}$ Adaption. 


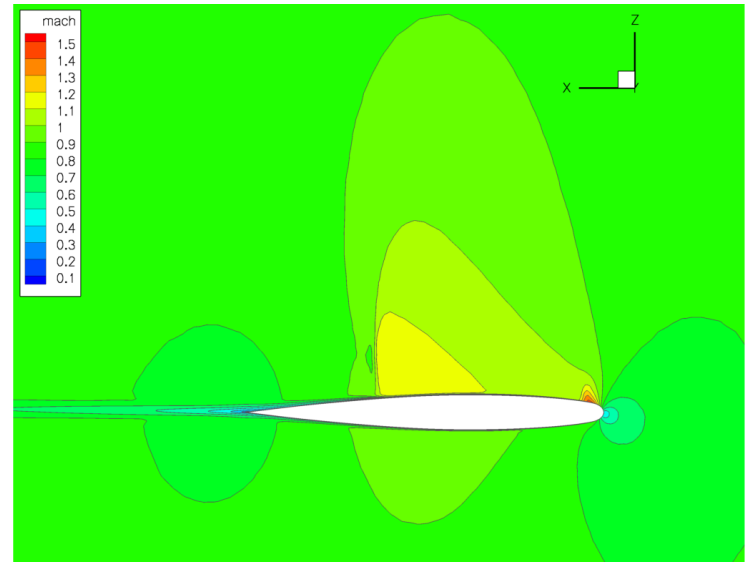

(a) GGNS, $y / b=0.2$.

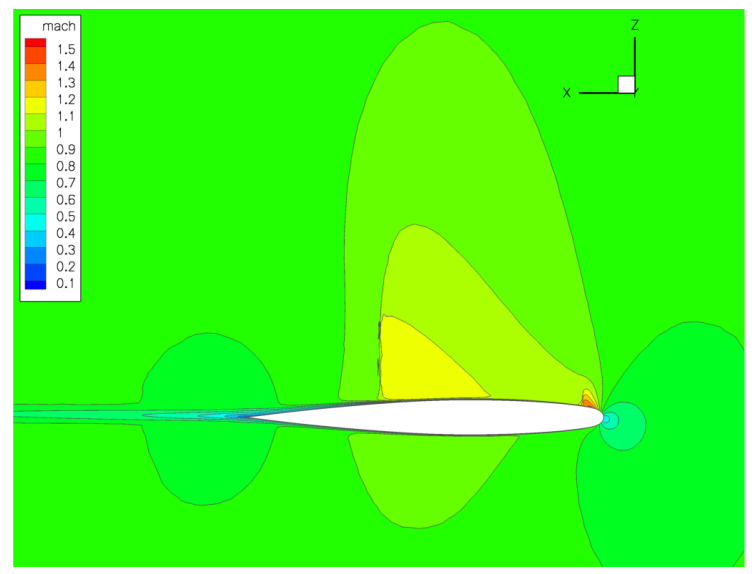

(c) FUN3D-FV, alternative wall distance, $y / b=0.2$.

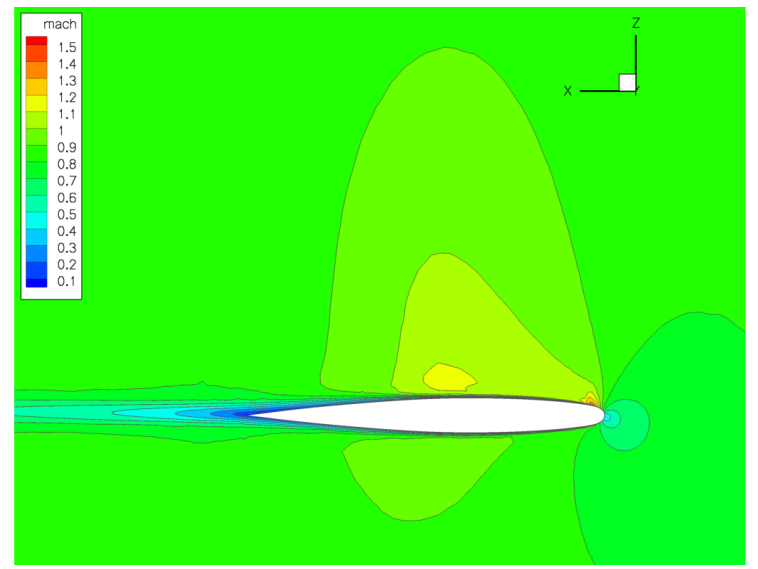

(e) FUN3D-FV, standard wall distance, $y / b=0.2$.

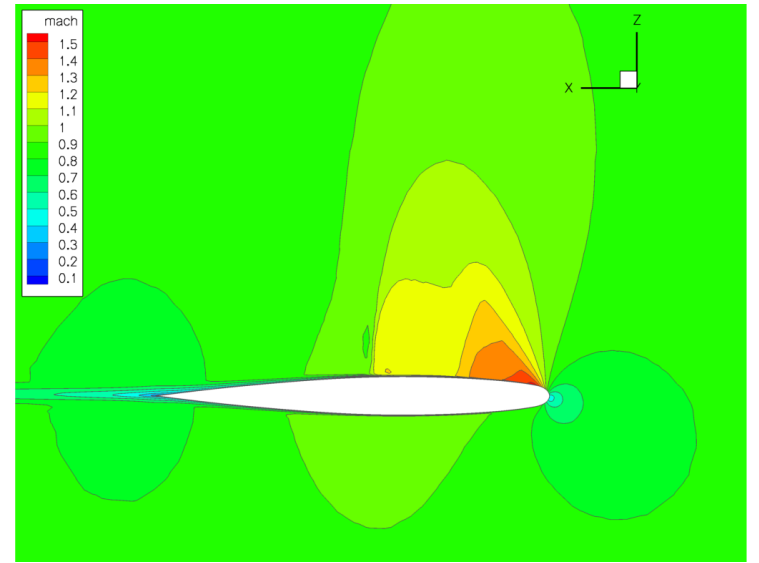

(b) GGNS, $y / b=0.7$.

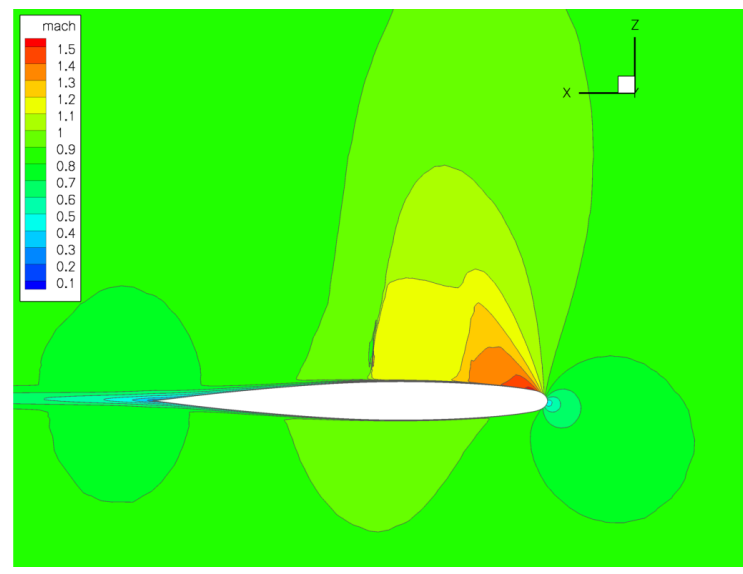

(d) FUN3D-FV, alternative wall distance, $y / b=0.7$.

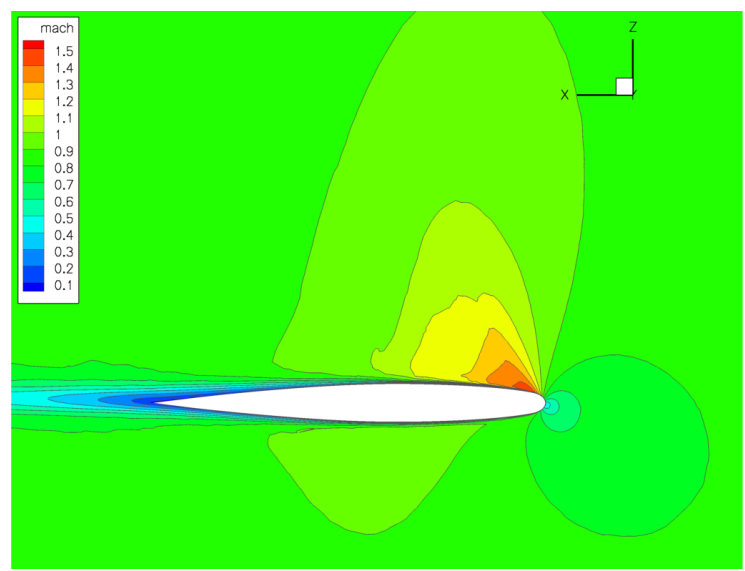

(f) FUN3D-FV, standard wall distance, $y / b=0.7$.

Fig. 13 Constant span slices of the iteration 17 grid of GGNS+EPIC-ICS $L_{p=4}$ Adaption. 


\section{Metric Conformity}

The generation of the adapted grids is a key step in the grid adaptation process, as generating high quality grids is essential to reach the prescribed error target. Only a few grid adaptation packages are actually able to output such curved boundary conforming grids adapted to a highly anisotropic metric field in 3D. The codes considered in this study are grid adapters, that output the adapted grid from an initial grid through a series of local modifications and optimizations. Different approaches are followed by the different codes as described in Section [I] Understanding how this implementation differences influence the final results is important.

Previously, simple geometries and analytic metrics were considered [13]. Both the geometry and metric complexity has increased in this study. These metrics and geometries are extracted from the RANS adaptation cases in this paper. In this study, we considered snapshots from the adaptation process involving FUN3D-FV and refine, both for the Hemisphere Cylinder case and the ONERA M6 wing case. The target complexity was continuously increased during the iterative Mach $L_{p=2}$ adaptation process. The FUN3D-FV and refine adaptation trajectories are representative of the intermediate grids and metrics of the integrated adaptive procedure trajectories. Grid/metric pairs were extracted from these trajectories, where the metric is formed from solution computed on the paired grid. Each of the adaptation codes then adapts a new grid from this pair, guided by the given metric field.

The properties of the grids are evaluated using the metric interpolated by each adaptation code from the provided input grid. Two local criteria of metric satisfaction are employed for descriptive statistics. First, the edge-length criterion as presented by Park et al. [12] is used. The edge length formula is based on an assumption that the logarithm of desired length varies linearly along the edge [40] and is defined by

$$
\begin{gathered}
L_{e}= \begin{cases}\frac{L_{a}-L_{b}}{\log \left(L_{a} / L_{b}\right)} & \left|L_{a}-L_{b}\right|>0.001 \\
\frac{L_{a}+L_{b}}{2} & \text { else }\end{cases} \\
L_{a}=\left(v_{e}^{T} M_{a} v_{e}\right)^{\frac{1}{2}}, L_{b}=\left(v_{e}^{T} M_{b} v_{e}\right)^{\frac{1}{2}}
\end{gathered}
$$

An ideal edge has a metric length of one in a unit grid. Second, element shape is examined using the mean ratio formula for tetrahedra $Q_{K}$ where $K$ is a tetrahedron, $|K|$ is its volume, $v_{e}$ is the vector along one of its edges $e$, and $|\hat{K}|$ is the volume of a tetrahedron with unit edge lengths. $M_{\max }$ is a single metric tensor being used to measure the whole tetrahedron. In this case, we choose $M_{\max }$ as the adjacent vertex metric with largest determinant,

$$
\begin{gathered}
Q_{K}=\frac{\left(\frac{|K| \operatorname{det}\left(M_{\max }\right)^{\frac{1}{2}}}{|\hat{K}|}\right)^{\frac{2}{3}}}{\frac{1}{6} \sum_{e \in K} v_{e}^{T} M_{\max } v_{e}}, \\
M_{\max }=\underset{M_{v}, v \in K}{\arg \max \operatorname{det} M_{v} .}
\end{gathered}
$$

An ideal element has a mean ratio of one. We also consider the global criterion of number of elements, as the main purpose of solution-based adaptivity is to minimize the number of degrees of freedom while maximizing accuracy.

The distribution of these quantities is represented on histograms both in linear and logarithmic scales. The histograms in linear scale show that all the considered codes output grids where the edge lengths are clustered around 1 and mean ratio is clustered near 1 . The quality of the grid is judged depending on the width of the distribution, and the tails, which represents undesirable elements (too large, too small, poorly shaped). The histograms in logarithmic scale offer a better view of the tails of the distributions, and allow us to spot more precisely the number of low shape measure elements. A normalized count is used for the vertical axis, which is the number of members of a bin divided by the width of the bin and the total number of members of all bins. The integral under the histogram is equal to one with this normalization, which models a continuous probability density function. The bins are sized logarithmically to better resolve the tails. The size of the bins impacts the normalized count, particularly for small counts. The discrete levels in the tails of the logarithmic plots are due to small member counts. 


\section{A. Hemisphere Cylinder Metric Conformity}

Two versions of this grid/metric pair are taken at different iterations of the adaptation process. The first metric has a target complexity of 30,000, and the second metric has a larger complexity of 100,000. The cases will be respectively referred to as hsc-30k and hsc-100k. While the initial and target grids are relatively coarse in the first case and present relatively low anisotropic ratios, the prescribed anisotropy is significantly greater in the second case. This second case is more challenging due to the small boundary layer metric normal the model.

Histograms of edge lengths and mean ratios in linear scale for the hsc-30k case are provided in Fig. 14. They show that for all considered codes, the resulting shape measure distribution is centered about 1 , which is the ideal shape measure, both in terms of edge lengths and mean ratio. The distribution evaluated on the input grid is shown in black. For all codes, the distribution is more narrow than the input metric, which conveys that a large majority of elements have excellent shape measures. Omega_h, refine and Feflo.a have very similar edge length distributions, while Pragmatic tends to have longer edges than the others. EPIC-ICS has a bimodal distribution and EPIC-ICSM has the highest peak. In terms of mean ratio, the improvement from the initial grid is very clear for all codes, which all have similar distributions.

The logarithmic scale histograms in Fig. 15] show better some key differences between the codes. While Feflo.a has the narrowest distribution, it also has more low mean ratio elements. Conversely, Pragmatic and Omega_h have wider distributions but fewer low mean ratio elements.

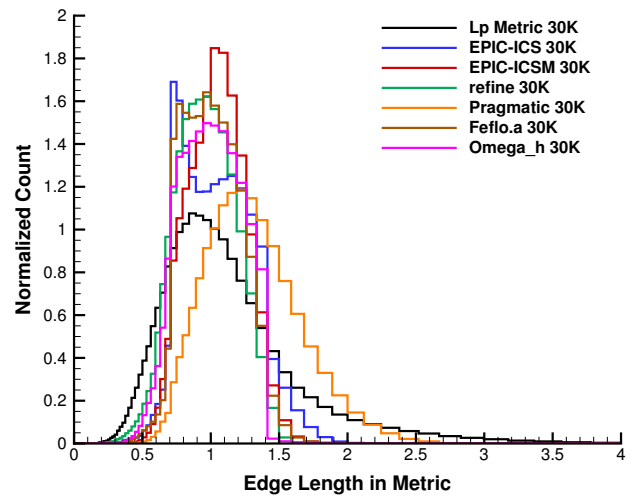

(a) Edge length in metric.

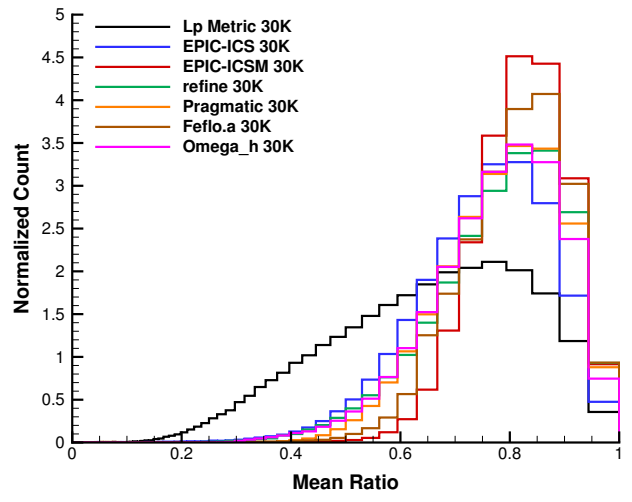

(b) Mean ratio.

Fig. 14 Hemisphere Cylinder metric conformity for 30K complexity.

The hsc-100k case exhibits similar trends as the hsc-30k case. The histograms are displayed in Fig. 16 and Fig. 17 One can notice that the initial grid was better converged than the hsc-30k one, and the improvements both in terms of edge lengths and mean ratio are smaller. In terms of edges lengths, EPIC-ICS still exhibits a flat distribution, while the peak of the distribution for Pragmatic is slightly above 1. Feflo.a and refine have a nicely shaped distribution centered about 1, but Feflo.a has significantly more very small or very long edges. In terms of mean ratio, all codes have a few low quality elements, which was expected due to the small grid sizes adjacent to the boundary. Pragmatic has significantly fewer mid-to-low mean ratio elements, while the worst mean ratio of Omega_h is only 0.15 , versus lower than 0.05 for the others. 


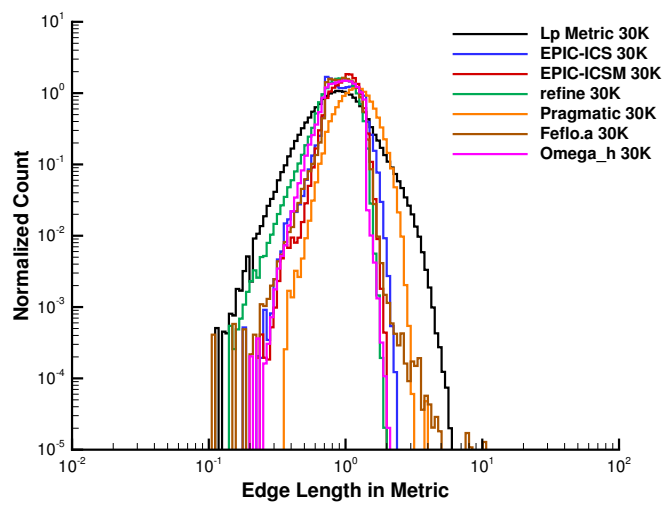

(a) Edge length in metric.

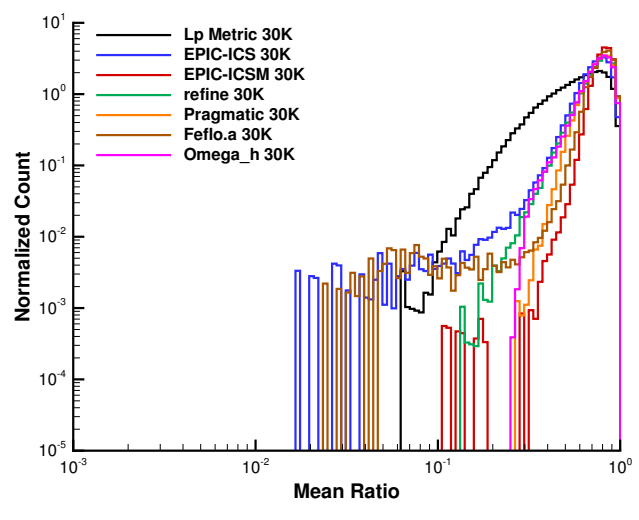

(b) Mean ratio.

Fig. 15 Hemisphere Cylinder metric conformity for $30 \mathrm{~K}$ complexity (log scale).

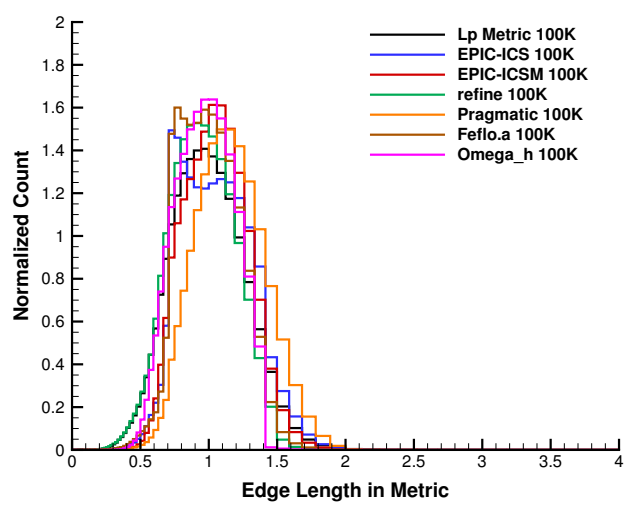

(a) Edge length in metric.

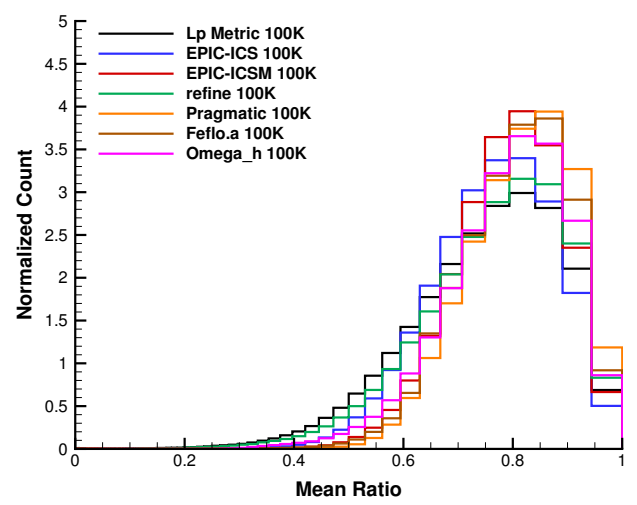

(b) Mean ratio.

Fig. 16 Hemisphere Cylinder metric conformity for 100K complexity.

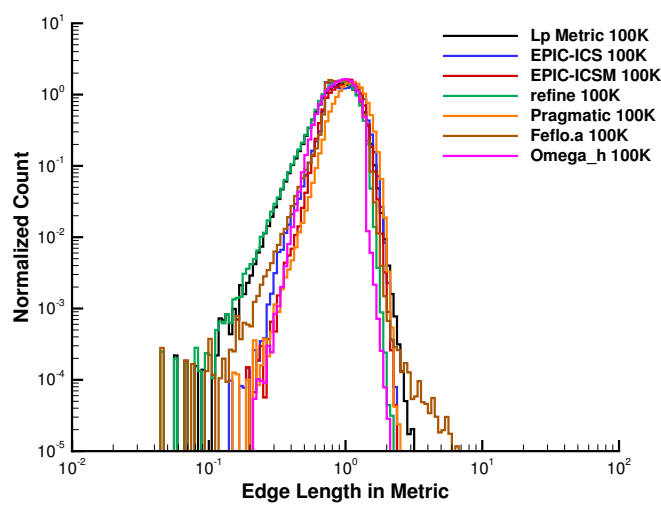

(a) Edge length in metric.

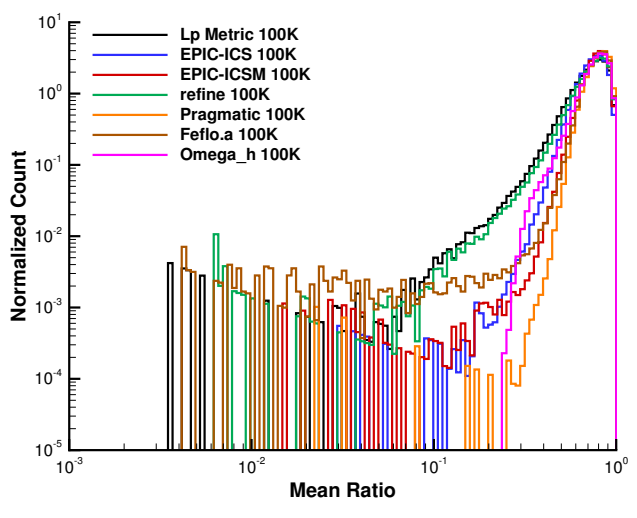

(b) Mean ratio.

Fig. 17 Hemisphere Cylinder metric conformity for $100 \mathrm{~K}$ complexity (log scale). 


\section{B. ONERA M6 Metric Conformity}

Grid/metric pairs from the ONERA M6 wing FUN3D-FV+refine Mach $L_{p=2}$ continuous refinement trajectory are also adapted. Two versions of this case are considered, with metric complexities of 30,000 and 100,000. The cases will be respectively referred to as m6-30k and m6-100k. The geometry of this case is more complex than for the hemisphere cylinder, and the very thin anisotropic boundary layer is expected to be a challenge, notably close to the tip and the trailing edge of the wing.

Grid histograms in linear and logarithmic scale for the m6-30k case are displayed in Fig. 18 and Fig. 19, respectively. The same kind of edge length distributions as in the hemisphere cylinder case can be found; however, more small edges appear for all codes. A closer look shows that EPIC-ICS produces a bimodal distribution, whereas Pragmatic produces slightly longer edges. In terms of mean ratio, the initial grid is not well converged yet, and presents many low quality elements. All codes perform equally well at narrowing the mean ratio distribution around 1; however, all the codes have a long tail of low mean ratio elements. With regards to this, EPIC-ICSM has the most high mean ratio elements and the smallest mean ratio.

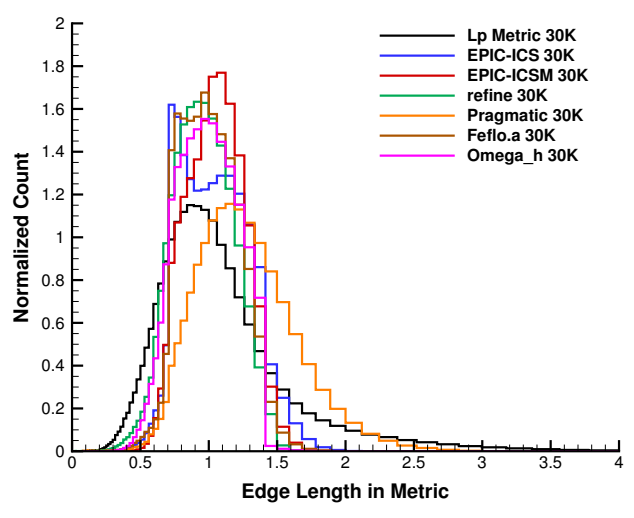

(a) Edge length in metric.

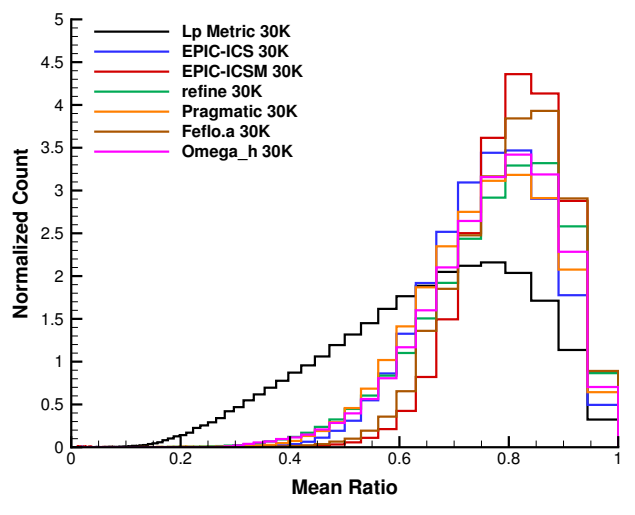

(b) Mean ratio.

Fig. 18 ONERA M6 metric conformity for 30K complexity.

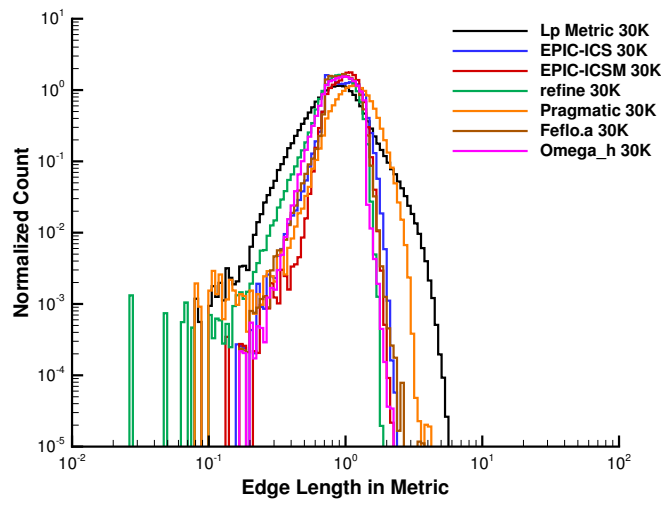

(a) Edge length in metric.

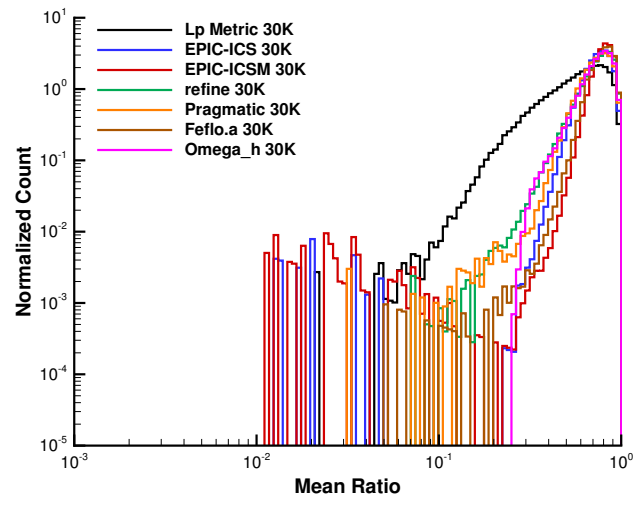

(b) Mean ratio.

Fig. 19 ONERA M6 metric conformity for 30K complexity (log scale).

For the bigger m6-100k case, the histograms are displayed in Fig.20 and Fig. 21 for the linear and logarithmic scales respectively. The initial grid is, like in the hemisphere cylinder case, already reasonably good, and the adaptation codes are expected to perform incremental optimization. This can prove to be delicate, as there is little room for maneuver 
close to the surface of the wing. Edge length distribution are very similar to the other cases, EPIC-ICS still exhibits its bimodal distribution and Pragmatic has slightly longer edges, but the trail of extreme sizes is bigger for Feflo.a and refine. The difference between the codes is more visible in terms of the mean ratio. All of the codes have some low mean ratio elements. The distribution of Pragmatic has a higher slope in the logarithmic scale, which results in fewer mid to low mean ratio elements. Despite a very small number of very low mean ratio elements, refine also performs well in limiting the number of low mean ratio elements. At the other end of the spectrum, Feflo.a creates a relatively large number of low mean ratio elements and a greater number of long edges in the metric than the input grid.

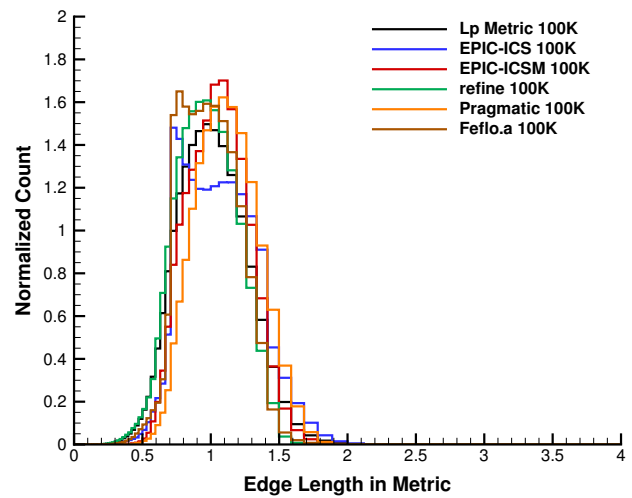

(a) Edge length in metric.

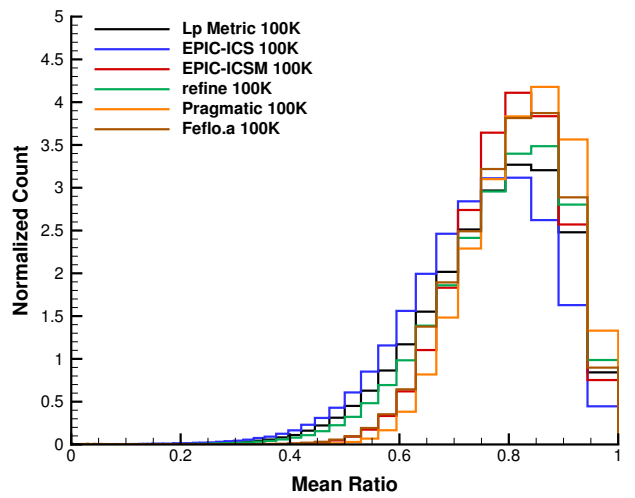

(b) Mean ratio.

Fig. 20 ONERA M6 metric conformity for 100K complexity.

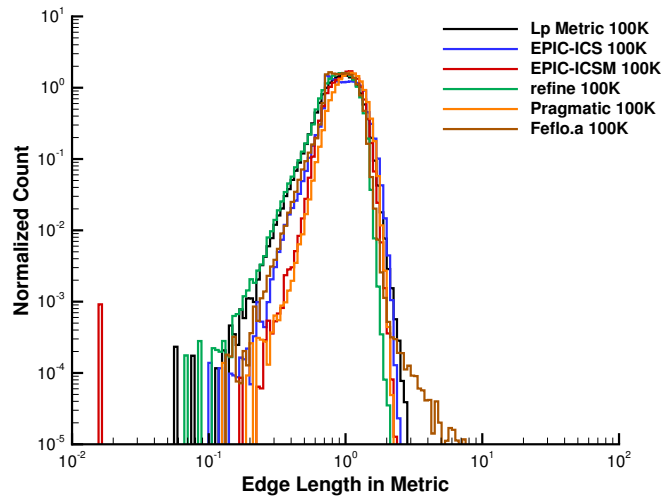

(a) Edge length in metric.

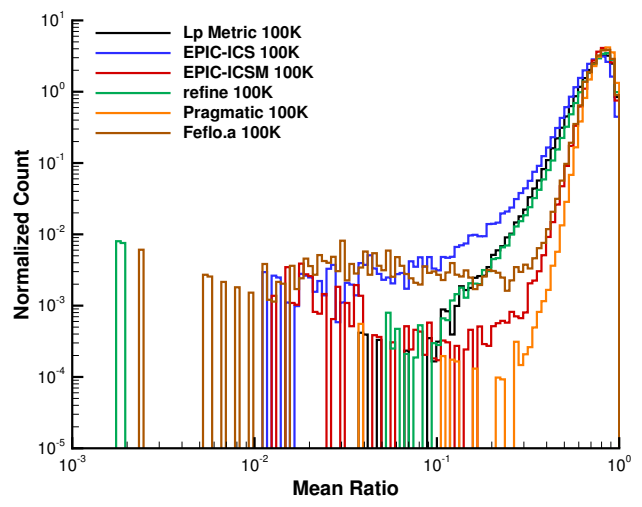

(b) Mean ratio.

Fig. 21 ONERA M6 metric conformity for 100K complexity (log scale).

\section{Conclusions}

Turbulent flow (RANS with SA turbulence model) adaptive simulations are conducted with refinement to curved geometry. The GGNS+EPIC-ICS adaptation to control the $L_{p}$ norm of Mach number showed forces and moment that converged toward fine, uniformly-refined, fixed-grids for the Alternative Hemisphere Cylinder and ONERA M6 cases. The metric for this error estimate can be readily constructed from a reconstructed Hessian of Mach number and linear algebra. Other adaptive processes, such as FUN3D-FV+refine and Wolf+Feflo.a, converged slower toward the uniformly-refined, fixed-grids for this metric. This overall difference could be a result of differences in the flow solver 
discretization (finite-element versus finite-volume), metric construction, adaptive grid mechanics, or a combination of factors. An assumption violation in the standard FUN3D-FV wall distance calculation negatively impacted the FUN3D-FV+refine calculations on adapted grids. The adapted-grid FUN3D-FV results with the alternative wall distance method were more consistent with fixed-grid results and other integrated adaptive processes.

Output-based metrics that control error estimates of lift and drag converged faster than the simple Mach $L_{p}$ metric. Functional convergence rates of the $L_{p=2}$ norm was not consistently better or worse than $L_{p=4}$ across implementations. Adaptive grids from the GGNS+EPIC-ICS integrated adaptive process where evaluated with FUN3D-FV and Wolf flow solvers. The FUN3D-FV and Wolf calculations on the GGNS+EPIC-ICS grids were closer to the fine, fixed-grids than calculations within the FUN3D-FV+refine and Wolf+Feflo.a integrated adaptation processes. The impact of wall distance calculation method was shown on the FUN3D-FV solutions. The standard wall distance method increased the thickness of the boundary layer and reduced the acceleration of the Mach number over the upper surface of the ONERA M6.

Metric conformity was studied on example grid/metric pairs to isolate the adaptive grid mechanics from other elements of integrated solution adaptation. This also allows grid mechanics to be evaluated that are not directly integrated with a flow solver and metric construction method. Comparison studies show that all the considered adaptive grid mechanics, despite different approaches in the grid optimization process, can output boundary and metric conforming adapted grids. They all output grids with edge lengths near unity in the metric and very few low mean ratio elements. A compromise may be present between the width of the mean ratio distribution and its tail, i.e., the number of very low mean ratio elements. Further analysis could confirm the location of the low mean ratio elements, and determine the sensitivity of the codes to undesirable elements in the initial grid.

Further progress in understanding the variation in adaptive grid trajectories of forces and moment will require further decomposition of the integrated grid adaptation processes. Fortunately, the group has adopted interchange formats and conventions for grids, metrics, and geometry. The consistent metric conformity results for all five adaptive codes indicate that the differences may be isolated to the metric construction or the flow solver. This investigation should be extended to the individual steps used to assemble the metric to further understand implementation details. For example, the three integrated grid adaptation processes used different Hessian recovery methods.

The Evaluation of RANS Solvers on Benchmark Aerodynamic Flows AIAA Special Sessions use uniformlyrefined, fixed grids with a significantly higher element orthogonality than the unstructured grids created in this study. Documenting the impact of discretization on a series of unstructured adaptive grids from the same trajectory could help to define improved metric or grid requirements. If orthogonality is shown to be critical, Michal et al. [47] inserted a semistructured prismatic boundary layer and Loseille [30] provides a method that encourages metric-orthogonal elements. More research is required to definitively show the impacts of orthogonality in the context of adaptive grid methods.

Ibanez et al. [13] and Park et al. [10] enumerate the open items that remain for future work. Studying more realistic and complicated CAD models with the possibility of missing topology, gaps larger than the required mesh size, and highly skewed surface parameterizations would increase the robustness of the adaptive grid mechanics. Parallel execution would permit larger grid sizes and faster execution. The execution time to a specified accuracy should be compared to fixed-grid methods to demonstrate practical utility and encourage adaptive grids to be the default approach. Error estimation and metric formation should be studied for multiple outputs and time-accurate simulations.

This study documents a clear improvement in five grid adaptation mechanic implementations. A marked increase in the complexity of the flow physics, adaptive metric, and geometry is attempted beyond Park et al. [12] and Ibanez et al. [13]. A quick estimate indicates that $O(1000)$ valid, metric-conforming, and boundary-conforming adaptive grids were formed, where approximately 30 adaptive trajectories had 30-50 grids. Multiple implementations of grid adaptation mechanics attained metric conformity as inferred by edge length and mean ratio descriptive statistics on a solution based metric. This effort demonstrates progress toward CFD Vision 2030 [11]. A number of the time line elements proposed by Park et al. [10] have been demonstrated. This work provides a benchmark for verifying the $L_{p}$ metric in integrated adaptive grid tools. These verified processes will set the stage for the infusion of solution interpolation error and ultimately output error controlled RANS simulations into production CFD workflows.

\section{Acknowledgements}

The authors would like to thank Kyle Anderson and Marshall Galbraith for discovering the assumption violation in the FUN3D-FV wall distance calculation for adapted grids. Matt O'Connell provided a wall distance implementation that is accurate for adapted grids. Cameron Druyor, Kyle Thompson, and Bil Kleb provided feedback that improved this 
manuscript. This work was partially funded under the embedded CSE programme of the ARCHER UK National Supercomputing Service (http://www.archer.ac.uk) and partially supported by the Transformational Tools and Technologies

(TTT) Project of the NASA Transformative Aeronautics Concepts Program (TACP).

\section{References}

[1] Mavriplis, D. J., Vassberg, J. C., Tinoco, E. N., Mani, M., Brodersen, O. P., Eisfeld, B., Wahls, R. A., Morrison, J. H., Zickuhr, T., Levy, D., and Murayama, M., "Grid Quality and Resolution Issues from the Drag Prediction Workshop Series," AIAA Journal of Aircraft, Vol. 46, No. 3, 2009, pp. 935-950. doi $10.2514 / 1.39201$.

[2] Levy, D. W., Laflin, K. R., Tinoco, E. N., Vassberg, J. C., Mani, M., Rider, B., Rumsey, C. L., Wahls, R. A., Morrison, J. H., Brodersen, O. P., Crippa, S., Mavriplis, D. J., and Murayama, M., "Summary of Data from the Fifth Computational Fluid Dynamics Drag Prediction Workshop,” AIAA Journal of Aircraft, Vol. 51, No. 4, 2014, pp. 1194-1213. doi 10.2514/1.C032389

[3] Morrison, J. H., "Statistical Analysis of the Fifth Drag Prediction Workshop Computational Fluid Dynamics Solutions," AIAA Journal of Aircraft, Vol. 51, No. 4, 2014, pp. 1214-1222. doi 10.2514/1.C032736

[4] Diskin, B., and Thomas, J. L., "Introduction: Evaluation of RANS Solvers on Benchmark Aerodynamic Flows," AIAA Journal, Vol. 54, No. 9, 2016, pp. 2561-2562. doi: $10.2514 / 1 . J 054642$

[5] Diskin, B., Thomas, J. L., Rumsey, C. L., and Schwöppe, A., "Grid-Convergence of Reynolds-Averaged Navier-Stokes Solutions for Benchmark Flows in Two Dimensions,” AIAA Journal, Vol. 54, No. 9, 2016, pp. 2563-2588. doi 10.2514/1.J054555

[6] Diskin, B., Thomas, J. L., Pandya, M. J., and Rumsey, C. L., "Reference Solutions for Benchmark Turbulent Flows in Three Dimensions," AIAA Paper 2016-858, 2016.

[7] Rumsey, C. L., "Recent Developments on the Turbulence Modeling Resource Website,” AIAA Paper 2015-2927, 2015.

[8] Spalart, P. R., and Allmaras, S. R., "A One-Equation Turbulence Model for Aerodynamic Flows," La Recherche Aérospatiale, Vol. 1, 1994, pp. 5-21.

[9] Alauzet, F., and Loseille, A., "A Decade of Progress on Anisotropic Mesh Adaptation for Computational Fluid Dynamics," Computer-Aided Design, Vol. 72, 2016, pp. 13-39. doi 10.1016/j.cad.2015.09.005, 23rd International Meshing Roundtable Special Issue: Advances in Mesh Generation.

[10] Park, M. A., Krakos, J. A., Michal, T., Loseille, A., and Alonso, J. J., "Unstructured Grid Adaptation: Status, Potential Impacts, and Recommended Investments Toward CFD Vision 2030,” AIAA Paper 2016-3323, 2016.

[11] Slotnick, J., Khodadoust, A., Alonso, J., Darmofal, D., Gropp, W., Lurie, E., and Mavriplis, D., "CFD Vision 2030 Study: A Path to Revolutionary Computational Aerosciences,” NASA CR-2014-218178, Langley Research Center, Mar. 2014. doi $2060 / 20140003093$

[12] Park, M. A., Loseille, A., Krakos, J. A., and Michal, T., "Comparing Anisotropic Output-Based Grid Adaptation Methods by Decomposition," AIAA Paper 2015-2292, 2015.

[13] Ibanez, D., Barral, N., Krakos, J., Loseille, A., Michal, T., and Park, M., "First Benchmark of the Unstructured Grid Adaptation Working Group," Procedia Engineering, Vol. 203, 2017, pp. 154-166. doi 10.1016/j.proeng.2017.09.800 26th International Meshing Roundtable, IMR26, 18-21 September 2017, Barcelona, Spain.

[14] Alauzet, F., and Loseille, A., "High-Order Sonic Boom Modeling Based on Adaptive Methods," Journal of Computational Physics, Vol. 229, No. 3, 2010, pp. 561-593. doi 10.1016/j.jcp.2009.09.020

[15] Haimes, R., and Drela, M., "On The Construction of Aircraft Conceptual Geometry for High-Fidelity Analysis and Design," AIAA Paper 2012-683, 2013.

[16] Loseille, A., and Alauzet, F., “Continuous Mesh Framework Part I: Well-Posed Continuous Interpolation Error,” SIAM Journal on Numerical Analysis, Vol. 49, No. 1, 2011, pp. 38-60. doi:10.1137/090754078

[17] Michal, T., and Krakos, J., “Anisotropic Mesh Adaptation Through Edge Primitive Operations,” AIAA Paper $2012-159,2012$.

[18] Alauzet, F., “A Changing-Topology Moving Mesh Technique for Large Displacements,” Engineering with Computers, Vol. 30, No. 2, 2014, pp. 175-200. doi $10.1007 / \mathrm{s} 00366-013-0340-\mathrm{Z}$ 
[19] Ibanez, D. A., "Conformal Mesh Adaptation on Heterogeneous Supercomputers," Ph.D. thesis, Rensselaer Polytechnic Institute, Nov. 2016.

[20] Ibanez, D., and Shephard, M., "Mesh Adaptation for Moving Objects on Shared Memory Hardware," 25th International Meshing Roundtable Research Notes, Sandia National Laboratories, 2016, pp. 1-5.

[21] Gorman, G. J., Rokos, G., Southern, J., and Kelly, P. H. J., "Thread-parallel anisotropic mesh adaptation,” New Challenges in Grid Generation and Adaptivity for Scientific Computing, Springer, 2015, pp. 113-137.

[22] Balay, S., Abhyankar, S., Adams, M. F., Brown, J., Brune, P., Buschelman, K., Dalcin, L., Eijkhout, V., Gropp, W. D., Kaushik, D., Knepley, M. G., McInnes, L. C., Rupp, K., Smith, B. F., Zampini, S., Zhang, H., and Zhang, H., "PETSc Users Manual," Tech. Rep. ANL-95/11 - Revision 3.8, Argonne National Laboratory, 2017. URL http://www . mcs . anl.gov/petsc

[23] Barral, N., Knepley, M. G., Lange, M., Piggott, M. D., and Gorman, G. J., "Anisotropic mesh adaptation in Firedrake with PETSc DMPlex," Sandia National Laboratories, 2016.

[24] Lipnikov, K., and Vassilevski, Y., "An adaptive algorithm for quasioptimal mesh generation,” Computational Mathematics and Mathematical Physics, Vol. 39, No. 9, 1999, pp. 1468-1486.

[25] Loseille, A., and Löhner, R., "Anisotropic Adaptive Simulations in Aerodynamics," AIAA Paper 2010-169, 2011.

[26] Loseille, A., "Chapter 10 - Unstructured Mesh Generation and Adaptation," Handbook of Numerical Methods for Hyperbolic Problems: Applied and Modern Issues, Handbook of Numerical Analysis, Vol. 18, edited by R. Abgrall and C.-W. Shu, Elsevier, 2017, pp. 263-302. doi:http://dx.doi.org/10.1016/bs.hna.2016.10.004

[27] Loseille, A., and Menier, V., "Serial and Parallel Mesh Modification Through a Unique Cavity-Based Primitive," Sandia National Laboratories, Springer International Publishing, 2014, pp. 541-558. doi 10.1007/978-3-319-02335-9_30.

[28] Loseille, A., Menier, V., and Alauzet, F., "Parallel Generation of Large-size Adapted Meshes," Procedia Engineering, Sandia National Laboratories, 2015, pp. 57-69. doi:10.1016/j.proeng.2015.10.122

[29] Loseille, A., and Löhner, R., "Robust Boundary Layer Mesh Generation,” Sandia National Laboratories, Springer Berlin Heidelberg, 2013, pp. 493-511. doi 10.1007/978-3-642-33573-0_29

[30] Loseille, A., "Metric-Orthogonal Anisotropic Mesh Generation," Procedia Engineering, Sandia National Laboratories, 2014, pp. 403-415. doi $10.1016 /$ j.proeng.2014.10.400.

[31] Allmaras, S. R., "Lagrange Multiplier Implementation of Dirichlet Boundary Conditions in Compressible Navier-Stokes Finite Element Methods," AIAA Paper 2005-4714, 2005.

[32] Kamenetskiy, D. S., Bussoletti, J. E., Hilmes, C. L., Venkatakrishnan, V., and Wigton, L. B., "Numerical Evidence of Multiple Solutions for the Reynolds-Averaged Navier-Stokes Equations,” AIAA Journal, Vol. 52, No. 8, 2014 , pp. $1686-1698$. doi $10.2514 / 1 . J 052676$

[33] Saad, Y., Iterative Methods for Sparse Linear Systems, $2^{\text {nd }}$ ed., Society for Industrial and Applied Mathematics, Philadelphia, PA, USA, 2003.

[34] Toro, E. F., Spruce, M., and Speares, W., "Restoration of the Contact Surface in the HLL-Riemann Solver," Shock Waves, Vol. 4, No. 1, 1994, pp. 25-34. doi $10.1007 /$ BF014146292

[35] Anderson, W. K., and Bonhaus, D. L., "An Implicit Upwind Algorithm for Computing Turbulent Flows on Unstructured Grids," Computers and Fluids, Vol. 23, No. 1, 1994, pp. 1-22. doi $10.1016 / 0045-7930(94) 90023-X$

[36] Biedron, R. T., Carlson, J.-R., Derlaga, J. M., Gnoffo, P. A., Hammond, D. P., Jones, W. T., Kleb, B., Lee-Rausch, E. M., Nielsen, E. J., Park, M. A., Rumsey, C. L., Thomas, J. L., and Wood, W. A., “FUN3D Manual: 13.2,” NASA TM-2016-219661, Langley Research Center, Aug. 2017. doi 2060/20170009600

[37] Roe, P. L., “Approximate Riemann Solvers, Parameter Vectors, and Difference Schemes," Journal of Computational Physics, Vol. 43, No. 2, 1981, pp. 357-372. doi 10.1016/0021-9991(81)90128-5

[38] Nielsen, E. J., Lu, J. C.-C., Park, M. A., and Darmofal, D. L., "An Implicit, Exact Dual Adjoint Solution Method for Turbulent Flows on Unstructured Grids," Computers and Fluids, Vol. 33, No. 9, 2004, pp. 1131-1155.

[39] Bonet, J., and Peraire, J., “An Alternating Digital Tree (ADT) Algorithm for 3D Geometric Searching and Intersection Problems," International Journal for Numerical Methods in Engineering, Vol. 31, No. 1, 1991, pp. 1-17. 
[40] Alauzet, F., "Size Gradation Control of Anisotropic Meshes," Finite Elements in Analysis and Design, Vol. 46, No. 1-2, 2010, pp. 181-202. doi $10.1016 /$ j.finel.2009.06.028

[41] Hsieh, T., "An Investigation of Separated Flow about a Hemisphere-Cylinder at 0- to 19-deg Incidence in the Mach Number Range from 0.6 to 1.5," Arnold Engineerig Development Center, Arnold Air Force Base AEDC-TR-76-112, Nov. 1976.

[42] Hsieh, T., "An Investigation of Separated Flow About a Hemisphere-Cylinder at Incidence in the Mach Number Range from 0.6 to 1.5," AIAA Paper 77-179, 1977.

[43] Groves, N. C., Huang, T. T., and Chang, M. S., "Geometric Characteristics of DARPA (Defense Advanced Research Projects Agency) SUBOFF Models (DTRC Model Numbers 5470 and 5471),” Tech. Rep. DTRC/SHDM-1298-01, David Taylor Research Center, Ship Hydromechanics Department, Mar. 1989. doi 2060/20160003613

[44] Advisory Group for Aerospace Research and Development, "Experimental Data Base for Computer Program Assessment: Report of the Fluid Dynamics Panel Working Group 04," NATO Research and Technology Organisation AGARD AR-138, Jan. 1979.

[45] Mayeur, J., Dumont, A., Destarac, D., and Gleize, V., "Reynolds-Averaged Navier-Stokes Simulations on NACA0012 and ONERA-M6 Wing with the ONERA elsA Solver," AIAA Journal, Vol. 54, No. 9, 2016, pp. 2671-2687. doi 10.2514/1.J054512

[46] Mayeur, J., Dumont, A., Destarac, D., and Gleize, V., "RANS Simulations on TMR 3D Test Cases with the ONERA elsA Flow Solver," AIAA Paper 2016-1357, 2016.

[47] Michal, T. R., Kamenetskiy, D. S., Krakos, J., Mani, M., Glasby, R. S., Erwin, T., and Stefanski, D., "Comparison of Fixed and Adaptive Unstructured Grid Results for Drag Prediction Workshop 6," AIAA Paper 2017-961, 2017. 